\title{
AN ADAPTIVE MODEL OF ASSET PRICE AND WEALTH DYNAMICS IN A MARKET WITH HETEROGENEOUS TRADING STRATEGIES
}

\author{
CARL CHIARELLA AND XUE-ZHONG HE \\ School of Finance and Economics \\ University of Technology, Sydney \\ PO Box 123 Broadway \\ NSW 2007, Australia
}

\begin{abstract}
This paper develops an adaptive model of asset price and wealth dynamics in a financial market with heterogeneous agents and examines the profitability of momentum and contrarian trading strategies. In order to characterize asset prices, wealth dynamics and rational adaptiveness arising from the interaction of heterogeneous agents with constant relative risk aversion (CRRA) utility, an adaptive discrete time equilibrium model in terms of return and wealth proportions (among heterogeneous representative agents) is established. Taking trend followers and contrarians as the main heterogeneous agents in the model, the profitability of momentum and contrarian trading strategies is analyzed. Our results show the capability of the model to characterize some of the existing evidence on many of the anomalies observed in financial markets, including the profitability of momentum trading strategies over short time intervals and of contrarian trading strategies over long time intervals, rational adaptiveness of agents, overconfidence and underreaction, overreaction and herd behavior, excess volatility, and volatility clustering.
\end{abstract}

Date: First Version: Dec.10, 2001; Latest Version: September 7, 2006.

Key words and phrases. Asset pricing, wealth dynamics, heterogeneity, adaptiveness, profitability, momentum trading strategies, contrarian trading strategies.

Acknowledgments. Financial support from the ARC under Discovery Grant DP0450526 is greatly acknowledged. 


\section{INTRODUCTION}

The traditional asset-pricing models - such as the capital asset pricing model (CAPM) of Sharpe (1964) and Lintner (1965), the arbitrage pricing theory (APT) of Ross (1976), or the intertemporal capital asset pricing model (ICAPM) of Merton (1973) have as one of their important assumptions, investor homogeneity. In particular the paradigm of the representative agent assumes that all agents are homogeneous with regard to their preferences, their expectations and their investment strategies. However, as already argued by Keynes in the 1930s, agents do not have sufficient knowledge of the structure of the economy to form correct mathematical expectations that would be held by all agents.

The other important paradigm underpinning these models, the efficient market hypothesis $(\mathrm{EMH})$, maintains that the current price contains all available information and past prices cannot help in predicting future prices. However there is evidence that markets are not always efficient and there are periods when real data show significantly higher autocorrelation of returns than would be expected under EMH. Over the last decade, a large volume of empirical work (e.g., Capaul et al (1993), Jegadeesh and Titman (1993, 2001), Asnee (1997), Rouwenhorst (1998), Arshanapali et al (1998), Fama and French (1998), Moskowitz and Grinblatt (1999), and Lee and Swaminathan (2000)) has documented a variety of ways in which asset returns can be predicted based on publicly available information and many of the results can be thought of as belonging to one of two broad categories of phenomena ${ }^{1}$. On the one hand, returns appear to exhibit continuation, or momentum, over short to medium time intervals, which may imply the profitability of momentum trading strategies over short to medium time intervals. On the other hand, there is also a tendency toward reversals over long time intervals, leading to possible profitability of contrarian strategies. The traditional models of finance theory seem to have difficulty in explaining this growing set of stylized facts. As a result, there is a growing dissatisfaction with (i) models of asset price dynamics based on the representative agent paradigm, as expressed for example by Kirman (1992), and (ii) the extreme informational assumptions of rational expectations.

In order to extend the traditional models of finance theory so as to accommodate some of the aforementioned stylized facts, a literature has developed over the last decade that involves some departure from the classical assumptions of strict rationality and unlimited computational capacity, and introduces heterogeneity and bounded rationality of agents. This strand of literature seeks to explain the existing evidence on many of the anomalies observed in financial markets as the result of the dynamic interaction of heterogeneous agents. In financial markets, individuals are imperfectly rational. They seek to learn about the market from their trading outcomes, as a result the market may fluctuate around the fully rational equilibrium. A number of recent models use this approach to characterize the interactions of heterogeneous agents in financial markets (e.g. Frankel and Froot (1987), Day and Huang (1990), Chiarella (1992), Lux (1995), Brock and Hommes (1997), (1998), Bullard and Duffy (1999), Chiarella and He (2003, 2002), Farmer (1999), Farmer and Lo (1999), Franke and Nesemann (1999), Lux and Marchesi (1999) LeBaron (2000), and Hommes (2001)). To avoid the constraints of analytical tractability, many of these authors use computer simulations to explore a wider range of economic settings. A general finding in many of

\footnotetext{
${ }^{1}$ A detailed discussion and references to the related empirical work is provided in Section 2.
} 
these studies is that long-horizon agents frequently do not drive short-horizon agents out of financial markets, and that populations of long- and short-horizon agents can create patterns of volatility and volume similar to actual empirical patterns.

Brock and Hommes $(1997,1998)$ propose to model economic and financial markets as an adaptive belief system (ABS), which is essentially an evolutionary competition among trading strategies. A key aspect of these models is that they exhibit expectations feedback and adaptiveness of agents. Agents adapt their beliefs over time by choosing from different predictors or expectations functions, based upon their past performance as measured by realized profits. Agents have the standard constant absolute risk aversion (CARA) utility function of the CAPM world but are boundedly rational, in the sense that they do not know the distribution of future returns. The evolutionary model generates endogenous price fluctuations with similar statistical properties to those observed in financial markets. The model of Brock and Hommes has been extended in Chiarella and $\mathrm{He}$ (2002) by allowing agents to have different risk attitudes and different expectation formation schemes for both first and second moments of the price distribution.

Because of the underlying CARA utility function, investors' optimal decisions depend only on the asset price and do not directly involve their wealth. The resulting separation of asset price and wealth dynamics greatly simplifies the analysis of the model. However, a consequence of this separation is that the model cannot generate the type of growing price process that is observed in the market. Levy, Levy and Solomon (1994) and Levy and Levy (1996) consider a more realistic model where investors' optimal decisions depend on their wealth (as a result of an underlying constant relative risk aversion (CRRA) utility function) and both price and wealth processes are intertwined and thus growing. Using numerical simulations and comparing the stock price dynamics in models with homogeneous and heterogeneous expectations, they conclude that the homogeneous expectations assumption leads to a highly inefficient market with periodic (and therefore predictable) booms and crashes while the introduction of heterogeneous expectations leads to much more realistic dynamics and more efficient markets.

Chiarella and He (2001) develop a theoretical model of interaction of portfolio decisions and wealth dynamics with heterogeneous agents having CRRA utility function. A growth equilibrium model of both the asset price and wealth is obtained. To characterize the interaction of heterogeneous agents in financial markets and conduct a theoretical analysis, stationary models in terms of return and wealth proportions (among different types of agents) are then developed. As a special case of the general heterogeneous model, these authors consider models of homogeneous agents and of two heterogeneous agents without switching of strategies. It is found that, in these cases, the heterogeneous model can have multiple steady states and the convergence to the steady states follows an optimal selection principle - the return and wealth proportions tend to the steady state which has relatively higher return. The model developed displays the volatility clustering of the returns and the essential characteristics of the standard asset price dynamics model of continuous time finance in that the asset price is fluctuating around a geometrically growing trend.

The aim of the current paper is twofold. First to establish an adaptive model of asset price and wealth dynamics in an economy of heterogeneous agents that extends the model in Chiarella and He (2001) to allow agents to switch amongst different types 
of trading strategies. Second to characterize the profitability of the two most popular trading strategies in real markets-momentum and contrarian trading strategies.

According to Brock and Hommes $(1997,1998)$ and the references cited therein, a financial market is an interaction of heterogeneous agents who adapt their beliefs from time to time. In our model, based on certain fitness measures, such as realized wealth, the agents are allowed to switch from one strategy to another from time to time. Consequently a model with adaptive beliefs is established where evolutionary dynamics across predictor choice is coupled with the dynamics of the endogenous variables.

Empirical studies provide some evidence that momentum trading (or trend following) strategies are more profitable over short time intervals, while contrarian trading strategies are more profitable over long time intervals (see for instance Capaul et al (1993), Jegadeesh and Titman (1993, 2001), Asnee (1997), Arshanapali et al (1998), Fama and French (1998), Rouwenhorst (1998), Moskowitz and Grinblatt (1999), Lee and Swaminathan (2000), and Levis and Liodakis (2001)). To characterize the profitability of momentum and contrarian trading strategies, a quasi-homogeneous model is introduced, in which agents use exactly the same trading strategies except for using different time horizons. Our results in general support the empirical findings on the profitability of momentum and contrarian trading strategies. In addition, the model also exhibits the various anomalies observed in financial markets, including, overconfidence and underreaction, overreaction and herd behavior, excess volatility, and volatility clustering.

This paper is organized as follows. Section 2 establishes an adaptive model of asset price and wealth dynamics with heterogeneous beliefs amongst agents. It is shown how the distributions of the wealth and population across heterogeneous agents are measured. As a simple case, a model of two types of agents is then considered in Section 3. To characterize the profitability of momentum and contrarian trading strategies, a quasi-homogeneous model is also introduced as a special case of the model of two types of agent in Section 3. The profitability of momentum and contrarian trading strategies is then analyzed in Sections 4 and 5, respectively. Section 6 concludes.

\section{Adaptive Model with Heterogeneous Agents}

This section is devoted to establishing an adaptive model of asset price and wealth dynamics with heterogeneous beliefs amongst agents. The model can been treated as a generalization and extension of some recent asset pricing models involving the interaction between heterogeneous agents, for example, Levy and Levy (1996), Barberis et al (1998), Brock and Hommes (1998), Daniel et al (1998), Hong and Stein (1999) and Chiarella and He (2001). The key characteristics of this modelling framework are the adaptiveness, the heterogeneity and the interaction of the economic agents. The heterogeneity is expressed in terms of different views on expectations of the distribution of future returns on the risky asset. The modelling framework of this paper extends that of the earlier cited works by focusing on the interaction of both asset price and wealth dynamics (Brock and Hommes (1998) considered only asset price dynamics) and by allowing a mechanism of adaptiveness of heterogeneous agents (Chiarella and $\mathrm{He}$ (2001) considered fixed proportions of heterogeneous agents). 
The framework of the adaptive model developed here is similar to the one in Levy and Levy (1996) and Chiarella and He (2001). Our hypothetical financial market contains two investment choices: a stock (or index of stocks) and a bond. The bond is assumed to be a risk free asset and the stock is a risky asset. The model is developed in the discrete time setting of standard portfolio theory in that agents are allowed to revise their portfolios over each time interval, the new element being the heterogeneity of agents and the way in which they form expectations on the return distributions.

The use of CARA utility functions has been standard in much of asset pricing theory. It has the characteristic of leading to demands that do not depend on the agents' wealth, but this dependence turns out to be quite crucial in developing a model exhibiting a growing price trend. A CRRA utility function is sufficient to capture the interdependence of price and wealth dynamics. The selection of logarithmic utility in the model developed here is based on a number of experimental and empirical studies, as summarized in Levy, Levy and Solomon (2000) that, "it is reasonable to assume decreasing absolute risk aversion (DARA) and constant relative risk aversion (CRRA)" (p.65). They show that the only utility function with DARA and CRRA property is the power utility function, among which, the logarithmic utility function is one of the special cases.

For the standard portfolio optimization problem, a model in terms of price and wealth is first established in this section. However it turns out that the resulting model is non-stationary in that both the price and wealth are growing processes. So, in order to reduce the growth model to a stationary model, the return on the risky asset and the wealth proportions (among heterogeneous investors), instead of price and wealth, are used as state variables. Based on a certain performance (or fitness) measures, an adaptive mechanism is finally introduced, leading to the general adaptive model. The final model includes the dynamics of both the asset price and wealth and it characterizes three important and related issues in the study of financial market: heterogeneity, adaptiveness, and interaction of agents.

\subsection{Notation. Denote}

$p_{t}$ : Price (ex dividend) per share of the risky asset at time $t$;

$y_{t}:$ Dividend at time $t$;

$R$ : $\quad$ Risk free return with risk free rate $r=R-1$;

$N$ : Total number of shares of the risky asset;

$H$ : Total number of investors;

$N_{i, t}$ : Number of shares acquired by agent $i$ at time $t$;

$W_{i, t}$ : Wealth of agent $i$ at time $t$

$W_{i, 0}$ : Initial wealth of agent $i$;

$\pi_{i, t}$ : Proportion of wealth of agent $i$ invested in the risky asset at time $t$;

$\rho_{t}$ : The return on the risky asset at period $t$. 
It is assumed that ${ }^{2}$ all the agents have the same attitude to risk with the same utility function $U(W)=\log (W)$. Following the above notation, the return on the risky asset at period $t$ is then defined by ${ }^{3}$

$$
\rho_{t}=\frac{p_{t}-p_{t-1}+y_{t}}{p_{t-1}}
$$

2.2. Portfolio Optimization Problem of Heterogeneous Agents. Following the standard portfolio optimization approach, the wealth of agent (or investor) $i$ at time period $t+1$ is given by

$$
\begin{aligned}
W_{i, t+1} & =\left(1-\pi_{i, t}\right) W_{i, t} R+\pi_{i, t} W_{i, t}\left(1+\rho_{t+1}\right) \\
& =W_{i, t}\left[R+\pi_{i, t}\left(\rho_{t+1}-r\right)\right] .
\end{aligned}
$$

As in Brock and Hommes (1998) and Levy and Levy (1996), a Walrasian scenario is used to derive the demand equation, that is each trader is viewed as a price taker and the market is viewed as finding (via the Walrasian auctioneer) the price $p_{t}$ that equates the sum of these demand schedules to the supply. That is, the agents treat the period $t$ price, $p_{t}$, as parametric when solving their optimisation problem to determine $\pi_{i, t}$. Denote by $F_{t}=\left\{p_{t-1}, \cdots ; y_{t}, y_{t-1}, \cdots\right\}$ the information $\operatorname{set}^{4}$ formed at time $t$. Let $E_{t}, V_{t}$ be the conditional expectation and variance, respectively, based on $F_{t}$, and $E_{i, t}, V_{i, t}$ be the "beliefs" of investor $i$ about the conditional expectation and variance. Then it follows from (2.2) that

$$
\begin{aligned}
E_{i, t}\left(W_{i, t+1}\right) & =W_{i, t}\left[R+\pi_{i, t}\left(E_{i, t}\left(\rho_{t+1}\right)-r\right)\right], \\
V_{i, t}\left(W_{i, t+1}\right) & =W_{i, t}^{2} \pi_{i, t}^{2} V_{i, t}\left(\rho_{t+1}\right) .
\end{aligned}
$$

Consider investor $i$, who faces a given price $p_{t}$, has wealth $W_{i, t}$ and believes that the asset return is conditionally normally distributed with mean $E_{i, t}\left(\rho_{t+1}\right)$ and variance $V_{i, t}\left(\rho_{t+1}\right)$. This investor chooses a proportion $\pi_{i, t}$ of his/her wealth to be invested in the risky asset so as to maximize the expected utility of the wealth at $t+1$, as given by

$$
\max _{\pi_{i, t}} E_{i, t}\left[U\left(W_{i, t+1}\right)\right] .
$$

It follows that ${ }^{5}$ the optimum investment proportion at time $t, \pi_{i, t}$ is given by

$$
\pi_{i, t}=\frac{E_{i, t}\left(\rho_{t+1}\right)-r}{V_{i, t}\left(\rho_{t+1}\right)}
$$

Heterogeneous beliefs are introduced via the assumption that

$$
E_{i, t}\left(\rho_{t+1}\right)=f_{i}\left(\rho_{t-1}, \cdots, \rho_{t-L_{i}}\right), \quad V_{i, t}\left(\rho_{t+1}\right)=g_{i}\left(\rho_{t-1}, \cdots, \rho_{t-L_{i}}\right)
$$

${ }^{2}$ To make the following analysis more tractable and transparent, the assumption that all agents have the same utility function $U(W)=\log (W)$ is maintained in this paper. However, the analysis can be generalized to the case of the utility functions that allow agents to have different risk coefficients, say, $U_{i}(W)=\left(W^{\gamma_{i}}-1\right) / \gamma_{i}$ with $0<\gamma_{i}<1$. As shown by Chiarella and He (2002), the dynamics generated by the difference in risk aversion coefficient is an interesting and important issue that for the present model is left for future work.

${ }^{3}$ The return can also be defined by the difference of logarithms of the prices. It is known that the difference between these two definition becomes smaller as the time interval is reduced (say, from monthly to weekly or daily).

${ }^{4}$ Because of the Walrasian scenario, the hypothetical price $p_{t}$ at time $t$ is included in the information set to determine the market clearing price. However, agents form their expectations by using the past prices up to time $t-1$.

${ }^{5}$ See Appendix A.1 in Chiarella and He (2001) for details. 
for $i=1, \cdots, H$, where $L_{i}$ are integers, $f_{i}, g_{i}$ are some deterministic functions which can differ across investors. Under this assumption, both $E_{i, t}\left(\rho_{t+1}\right)$ and $V_{i, t}\left(\rho_{t+1}\right)$ are functions of the past prices up to $t-1$, which in turn implies the optimum wealth proportion $\pi_{i, t}$, defined by (2.4), is a function of the history of the prices $\left(p_{t-1}, p_{t-2}, \cdots\right)^{6}$.

2.3. Market Clearing Equilibrium Price-A Growth Model. The optimum proportion of wealth invested in the risky asset, $\pi_{i, t}$, determines the number of shares at price $p_{t}$ that investor $i$ wishes to hold:

$$
N_{i, t}=\frac{\pi_{i, t} W_{i, t}}{p_{t}}
$$

Summing the demands of all agents gives the aggregate demand. The total number of shares in the market, denoted by $N$, is assumed to be fixed, and hence the market clearing equilibrium price $p_{t}$ is determined by

$$
\sum_{i=1}^{H} N_{i, t}=\sum_{i=1}^{H} \frac{\pi_{i, t} W_{i, t}}{p_{t}}=N,
$$

i.e.,

$$
\sum_{i=1}^{H} \pi_{i, t} W_{i, t}=N p_{t} .
$$

Thus, equations (2.2) and (2.6) show that, in this model, as in real markets, the equilibrium price $p_{t}$ and the wealth of investors, $W_{t} \equiv\left(W_{1, t}, \cdots, W_{H, t}\right)$, are determined simultaneously. The optimum demands of agents are functions of the price and their wealth. Also, as observed in financial markets, the model implies that both the price and the wealth are growing processes in general.

2.4. Population Distribution Measure. Now suppose all the agents can be grouped in terms of their conditional expectations of mean and variance of returns of the risky asset. That is, within a group, all the agents follow the same expectation schemes on the conditional mean and variance of the return $\rho_{t+1}$, and hence the optimum wealth proportion $\left(\pi_{i, t}\right)$ invested in the risky asset for the agents are the same. Assume all the agents can be grouped as $h$ types (or groups) and group $j$ has $\ell_{j, t}$ agents at time $t$ with $j=1, \cdots, h$, then $\ell_{1, t}+\cdots+\ell_{h, t}=H$. Denote by $n_{j, t}$ the proportion of the number of agents in group $j$, at time $t$, relative to the total number of the investors, $H$, that is, $n_{j, t}=\ell_{j, t} / H$, so that $n_{1, t}+\cdots+n_{h, t}=1$.

Some simple examples on return and wealth dynamics when proportions of different types of agents $n_{j, t}$ are fixed over time are given in Chiarella and He (2001). However, this is a highly simplified assumption and it would be more realistic to allow agents to adjust their beliefs from time to time, based on some performance or fitness measures (say, for example, the realized returns or errors, as in Brock and Hommes (1998)).

\footnotetext{
${ }^{6}$ In Levy and Levy (1996), the hypothetical price $p_{t}$ is included in the above conditional expectations on the return and variance. In this case, the market clearing price is solved implicitly and is much more involved mathematically. The approach adopted here is the standard one in deriving the price via the Walrasian scenario and also keeps the mathematical analysis tractable. A similar approach has been adopted in Brock and Hommes (1997), (1998) and Chiarella and He (2002). Of course other market clearing mechanisms are possible, e.g., a market-maker. It turns out that the type of market clearing mechanism used does affect the dynamics, as this point see Chiarella and He (2003).
} 
In this way, one can account for investor psychology and herd behavior ${ }^{7}$. As a consequence, the proportions of different types of agents become endogenous state variables. Therefore the vector $\left(n_{1, t}, n_{2, t}, \cdots, n_{h, t}\right)$ measures the population distribution among different types of heterogeneous agents. The change in the distribution over time can be used to measure herd behavior among heterogeneous agents, in particular, during highly volatile periods in financial markets.

2.5. Heterogeneous Representative Agents and Wealth Distribution Measure. By assuming the adaptiveness of agents' behavior, agents may switch among different groups from time to time. To track the wealth evolution of each individual agent is certainly an interesting and important issue, but is rather a difficult problem within the current framework. However, by introducing heterogeneous representative agents (HRAs), the model established here is capable of characterizing their performance in terms of wealth distribution over time. By HRAs, we mean that such agents can become a fraction so that the sum of the fractions of all those agents is equal to 1 . More precise construction of such HRAs is given as follows.

Assume that all agents are grouped into $h$ types (according to their beliefs) and group $j$ had $\ell_{j, t}(i=1,2, \cdots, h)$ agents at time $t$. Here $h$ is assumed to be fixed, while $\ell_{j, t}$ can vary from period to period. At time $t$, for agents within the group $j$, let $\bar{W}_{j, t}$ be the average wealth of agents within group $j$, so that $\ell_{j, t} \bar{W}_{j, t}$ gives the total wealth of group $j$. Denote $\bar{w}_{j, t}$ as the average wealth proportion of group $j$ relative to the total average wealth $\bar{W}_{t}$ at time $t$, that is,

$$
\bar{w}_{i, t}=\frac{\bar{W}_{i, t}}{\bar{W}_{t}}, \quad \text { with } \quad \bar{W}_{t}=\sum_{j=1}^{h} \bar{W}_{j, t} .
$$

Then the vector $\left(\bar{w}_{1, t}, \bar{w}_{2, t}, \cdots, \bar{w}_{h, t}\right)$ corresponds to the wealth proportion distribution among HRAs of different types, it measure the average wealth levels associated with different trading strategies.

2.6. Performance Measure, Population Evolution and Adaptiveness. Following Brock and Hommes (1997), (1998), a performance measure or fitness function, denoted $\left(\Phi_{1, t}, \cdots, \Phi_{h, t}\right)$, is publicly available to all agents. Based on the performance measure agents make a (boundedly) rational choice among the predictors. This results in the Adaptive Rational Equilibrium Dynamics, introduced by Brock and Hommes (1997), an evolutionary dynamics across predictor choice which is coupled to the dynamics of the endogenous variables. In the limit as the number of agents goes to infinity, the probability that an agent $j$ chooses trading strategy $j$ is given by the well known discrete choice model or 'Gibbs' probabilities ${ }^{8}$

$$
n_{j, t}=\exp \left[\beta\left(\Phi_{j, t-1}-C_{j}\right)\right] / Z_{t} \quad Z_{t}=\sum_{j=1}^{h} \exp \left[\beta\left(\Phi_{j, t-1}-C_{j}\right)\right],
$$

where $C_{j} \geq 0$ measures the cost of the strategy $j$ for $j=1,2, \cdots, h$.

The crucial feature of (2.8) is that the higher the fitness of trading strategy $j$, the more traders will select that strategy. The parameter $\beta$, called intensity of choice or

\footnotetext{
${ }^{7}$ See more discussion on this aspect in the next section.

${ }^{8}$ See Manski and McFadden (1981) and Anderson, de Palma and Thisse (1993)) for extensive discussion of discrete choice models and their applications in economics.
} 
switching intensity, plays an important role and can be used to characterize various psychological effects, such as overconfidence and underreaction, overreaction and herd behavior, as discussed by Hirshleifer (2001). On the one hand, when individuals are overconfident, they do not change their beliefs as much as would a rational Bayesian in the face of new evidence. This may result from either high cost in processing new information or individuals' reluctant to admit to having made a mistake (so the new evidence is under-weighted). Both overconfidence and underreaction can be partially captured by a small value of the switching intensity parameter $\beta$. In the extreme case when $\beta=0$, there is no switching among strategies and the populations of agents is evenly distributed across all trading strategies ${ }^{9}$. On the other hand, if the environment is volatile, or agents are less confident about their beliefs, there may be no dishonor in recognizing that different beliefs are called for and agents are more willing to switch to beliefs which generate better outcomes. This effect can be captured by a high value of the switching intensity parameter $\beta$. An increase in the switching intensity $\beta$ represents an increase in the degree of rationality with respect to evolutionary selection of trading strategies. In the extreme case when $\beta$ is very large (close to infinity), a large proportion of traders are willing to switch more quickly to successful trading strategies. In such a situation, market overreaction and herd behavior may be observed.

A natural performance measure or fitness function can be taken as a weighted average of the realized wealth return on the proportion invested in the risky asset among $h$ HRAs, given by

$$
\Phi_{j, t}=\phi_{j, t}+\gamma \Phi_{j, t-1}
$$

for $j=1, \cdots, h$, where $0 \leq \gamma \leq 1$ and

$$
\phi_{j, t}=\bar{\pi}_{j, t-1} \frac{\bar{W}_{j, t}-\bar{W}_{j, t-1}}{\bar{W}_{j, t-1}}=\bar{\pi}_{j, t-1}\left[r+\left(\rho_{t}-r\right) \bar{\pi}_{j, t-1}\right]
$$

is the realized wealth return invested in the risky asset in period $t$. Here $\gamma$ is a memory parameter measuring how strongly the past realized fitness is discounted for strategy selection, so that $\Phi_{j, t}$ may be interpreted as the accumulated discounted return on the proportion of wealth invested by group $j$ in the risky asset.

2.7. An Adaptive Model. The above growth model is rendered stationary by formulating it in terms of the risky asset return and the average wealth proportions among the investors, instead of the wealth $W_{t}$ and the stock price $p_{t}$. It should be made clear that the term "stationary" is not being used in the econometric sense of a stationary stochastic process. Rather the term is used to refer to a stationary dynamical system that has fixed points (as opposed to growing trends) as steady state solutions. It may well be that the dynamical system to be analyzed below generates time series that are non-stationary in the econometric sense; this will depend on both the local stability/instability properties of the dynamical system and how noise is processed by the nonlinear system. When such a steady state exists, following from (2.1), it generates a geometrically growing price process. The dynamical system describing the evolution of average wealth proportions of HRAs and risk asset return is given by the following proposition.

\footnotetext{
${ }^{9}$ See Chiarella and He (2001) for models with fixed, but not evenly distributed, population proportion among different types of trading strategies.
} 
Proposition 2.1. For group $i$, formed at time period $t-1$, the average wealth proportions at the next time period t evolve according to

$$
\bar{w}_{i, t}=\frac{\bar{w}_{i, t-1}\left[R+\left(\rho_{t}-r\right) \bar{\pi}_{i, t-1}\right]}{\sum_{j=1}^{h} \bar{w}_{j, t-1}\left[R+\left(\rho_{t}-r\right) \bar{\pi}_{j, t-1}\right]} \quad(i=1, \cdots, h)
$$

with return $\rho_{t}$ given by

$$
\rho_{t}=r+\frac{\sum_{i=1}^{h} \bar{w}_{i, t-1}\left[(1+r)\left(n_{i, t-1} \bar{\pi}_{i, t-1}-n_{i, t} \bar{\pi}_{i, t}\right)-\alpha_{t} n_{i, t-1} \bar{\pi}_{i, t-1}\right]}{\sum_{i=1}^{h} \bar{\pi}_{i, t-1} \bar{w}_{i, t-1}\left(n_{i, t} \bar{\pi}_{i, t}-n_{i, t-1}\right)},
$$

where $\alpha_{t}$ denotes the dividend yield defined by $\alpha_{t}=y_{t} / p_{t-1}$, and the population proportions $n_{j, t}$ evolve according to

$$
n_{i, t}=\exp \left[\beta\left(\Phi_{i, t-1}-C_{j}\right)\right] / Z_{t},
$$

in which the fitness functions are defined by

$$
\begin{aligned}
\Phi_{i, t} & =\phi_{i, t}+\gamma \Phi_{i, t-1} ; \quad 0 \leq \gamma \leq 1, \\
\phi_{i, t} & =\bar{\pi}_{i, t-1} \frac{\bar{W}_{i, t}-\bar{W}_{i, t-1}}{\bar{W}_{i, t-1}}=\bar{\pi}_{i, t-1}\left[r+\left(\rho_{t}-r\right) \bar{\pi}_{i, t-1}\right], \\
Z_{t} & =\sum_{i=1}^{h} \exp \left[\beta\left(\Phi_{i, t-1}-C_{i}\right)\right],
\end{aligned}
$$

and the constants $C_{i} \geq 0$ measure the cost of the strategy for $i=1,2, \cdots, h$.

Proof. See Appendix A.1.

Equations (2.9) and (2.10) constitute a difference equation system for $\bar{w}_{j, t}$ and $\rho_{t}$ whose order depends on the choice by agents of the $L_{j}$ at equation (2.5). It should be stressed that in the process of rendering the model stationary it has become necessary to reason in terms of the dividend yield $\left(\alpha_{t}\right)$ rather than the dividend $\left(y_{t}\right)$ directly. It is easy to see that, when $h \leq H, \ell_{j} \geq 1$ and $\beta=0$ for $j=1, \cdots, h$, Proposition 2.1 leads to the model in Chiarella and He (2001) with fixed proportion $n_{j, t}=n_{j}(j=1, \cdots, h)$ of heterogeneous agents.

2.8. Trading Strategies. The adaptive model established in Proposition 2.1 is incomplete unless the conditional expectations of agents on the mean and variance of returns are specified. Different trading strategies can be incorporated into this general adaptive model as indicated by equation (2.5). To illustrate various features of the model, only three simple, but well-documented, types of agents, termed fundamentalists, momentum traders and contrarians, is considered in this paper. ${ }^{11}$ Neither type is fully rational in the sense used in the rational expectations literature. The information on the dividends and realized prices is publicly available to all agent types.

\footnotetext{
${ }^{10}$ It is easy to check that $\rho_{t} \equiv r$ is a trivial solution. As a necessary condition for investing in the risky asset, it is assumed that $E\left(\rho_{t}\right)>r$.

${ }^{11}$ To simplify the analysis, we focus on the conditional mean estimation by assuming that subjective estimation of variance of all the agents' is given by a constant.
} 
2.8.1. Fundamental traders. The fundamentalists make forecasts on the risk premium level based on both public and their private information about future fundamentals. It is assumed that

$$
E_{F, t}\left(\rho_{t+1}\right)=r+\delta_{F},
$$

where $E_{F, t}$ denotes the fundamentalists' expected return on $\rho_{t+1}$ for the next period $t+1$ and $\delta_{F}$ is the risk premium estimated. ${ }^{12}$ That is, the fundamentalists believe that the excess conditional mean for the risky asset (from the risk-free rate) is given by the risk premium $\delta_{F}$ that they may have estimated from a detailed analysis of the risky asset (earnings reports, market prospects, political factors etc.) .

2.8.2. Momentum Traders. Momentum traders, in contrast to the fundamental traders, do condition on the past prices. Momentum, or positive feedback, trading has several possible motivations, one being that agents form expectations of future prices by extrapolating trends. They buy into price trends and exaggerate them, leading to overshooting. As a result there may appear excess volatility.

Empirical studies have given support to the view that momentum trading strategies yield significant profits over short time intervals (e.g. Asnee (1997), Jegadeesh and Titman (1993), (2001), Lee and Swaminathan (2000), Moskowitz and Grinblatt (1999) and Rouwenhorst (1998)). Although these results have been well accepted, the source of the profits and the interpretation of the evidence are widely debated. In addition, there does not exist in the literature a quantitative model to clarify and give theoretical support to such evidence. As a first step, this issue is discussed in the next section within the framework of the adaptive heterogeneous model outlined in Proposition 2.1.

For momentum traders, it is assumed in this paper that their forecasts are "simple" functions of the history of past returns. More precisely, it is assumed that

$$
E_{M, t}\left(\rho_{t+1}\right)=r+\delta_{M}+d_{M} \bar{\rho}_{M, t}, \quad \bar{\rho}_{M, t}=\frac{1}{L_{M}} \sum_{k=1}^{L_{M}} \rho_{t-k},
$$

where $E_{M, t}\left(\rho_{t+1}\right)$ denotes the expected return of momentum traders on $\rho_{t+1}$ for the next period $t+1$ and $\delta_{M}$ is their risk premium estimate and $d_{M}>0$ corresponds to the extrapolation rate of the momentum trading strategy. The integer $L_{M} \geq 1$ corresponds to the memory length of momentum traders. Equation (2.13) states that the expected excess return (above the risk-free rate) of momentum traders has two components: their estimated risk premium $\delta_{M}$ and trend extrapolation $d_{M} \bar{\rho}_{M, t}$, which is positively proportional to the moving average of the returns over the last $L_{M}$ time periods.

2.8.3. Contrarian Traders. The profitability of contrarian investment strategies is now a well-established empirical fact in the finance literature (see, for example, Levis and Liodakis (2001)). Empirical evidence suggests that over long time intervals, contrarian strategies generate significant abnormal returns (see, for example, Arshanapali et al (1998), Fama and French (1998), and Capaul et al (1993)). Some evidence has shown that overreaction can use aggregate stock market value measures such as dividend yield to predict future market returns, so that contrarian investment strategies are

\footnotetext{
${ }^{12} \mathrm{~A}$ constant risk premium is a simplified assumption. In practice, the risk premium may not necessarily be constant but could also be a function of the variance, for example.
} 
on average profitable. In spite of the apparent robustness of such strategies, the underlying rationale for their success remains a matter of lively debate in both academic and practitioner communities.

In the following section, the role of expectational errors in explaining the profitability of contrarian strategies is examined. For contrarian traders, it is assumed that

$$
E_{C, t}\left(\rho_{t+1}\right)=r+\delta_{C}-d_{C} \bar{\rho}_{C, t}, \quad \bar{\rho}_{C, t}=\frac{1}{L_{C}} \sum_{k=1}^{L_{C}} \rho_{t-k},
$$

where $E_{C, t}\left(\rho_{t+1}\right)$ denotes the expected return of contrarian agents on $\rho_{t+1}$ for the next period $t+1$ and $\delta_{C}$ is their estimated risk premium and $d_{C}>0$ corresponds to their extrapolation rate. The integer $L_{C} \geq 1$ corresponds to the memory length of contrarian agents. Equation (2.14) states that contrarian traders believe that the difference of excess conditional mean and the risk premium $\left[E_{C, t}\left(\rho_{t+1}\right)-r\right]-\delta_{C}$ is negatively proportional to the moving average of the returns over the last $L_{C}$ time periods.

In addition to the different trading strategies used by different types of traders, there are various other ways to introduce agent heterogeneity such as through different risk premia, extrapolation rates and memory lengths.

\section{An Adaptive Model of Two Types of Agents}

In the rest of this paper, the focus is on a simple model of just two types of agentsmomentum traders and contrarian traders. In this case, the adaptive model developed in Section 2 can be reduced to a simple form, as indicated below. To examine profitability of momentum and contrarian trading strategies over different time intervals, a special case of the model, termed the quasi-homogeneous model, is then considered. Detailed discussion on the dynamics of such quasi-homogeneous models, including profitability, herd behavior, price overshooting, statistical patterns of returns, is then undertaken in the subsequent sections.

3.1. Notation. Assume that there are only two different types trading strategies. Let $\bar{w}_{t}, \bar{n}_{t}$ be the difference of the average wealth proportions and population proportions of type 1 and type 2 agents; that is

$$
\bar{w}_{t}=\bar{w}_{1, t}-\bar{w}_{2, t}, \quad n_{t}=n_{1, t}-n_{2, t} .
$$

Then it follows from $\bar{w}_{1, t}+\bar{w}_{2, t}=1$ and $n_{1, t}+n_{2, t}=1$ so that

$$
\bar{w}_{1, t}=\frac{1+\bar{w}_{t}}{2}, \quad \bar{w}_{2, t}=\frac{1-\bar{w}_{t}}{2}
$$

and

$$
n_{1, t}=\frac{1+n_{t}}{2}, \quad n_{2, t}=\frac{1-n_{t}}{2} .
$$

Correspondingly, the adaptive model in Proposition 2.1 can be reduced to a simple form.

3.2. The Model for Two Types of Agents. In terms of the above notation, the adaptive model for two types of agents following different trading strategies assumes the form give by Proposition 3.1. 
Proposition 3.1. The difference of the average wealth proportions $\bar{w}_{t}$ evolves according to

$$
\bar{w}_{t+1}=\frac{f_{1}-f_{2}}{f_{1}+f_{2}}
$$

with return $\rho_{t}$ given by

$$
\rho_{t+1}=r+\frac{g_{11}+g_{12}}{g_{21}+g_{22}}
$$

where

$$
\begin{aligned}
f_{1} & =\left(1+\bar{w}_{t}\right)\left[1+r+\left(\rho_{t+1}-r\right) \bar{\pi}_{1, t}\right], \\
f_{2} & =\left(1-\bar{w}_{t}\right)\left[1+r+\left(\rho_{t+1}-r\right) \bar{\pi}_{2, t}\right], \\
g_{11} & =\left(1+\bar{w}_{t}\right)\left[\left(1+r-\alpha_{t+1}\right)\left(1+\bar{n}_{t}\right) \bar{\pi}_{1, t}-(1+r)\left(1+\bar{n}_{t+1}\right) \bar{\pi}_{1, t+1}\right], \\
g_{12} & =\left(1-\bar{w}_{t}\right)\left[\left(1+r-\alpha_{t+1}\right)\left(1-\bar{n}_{t}\right) \bar{\pi}_{1, t}-(1+r)\left(1-\bar{n}_{t+1}\right) \bar{\pi}_{2, t+1}\right], \\
g_{21} & =\left(1+\bar{w}_{t}\right) \bar{\pi}_{1, t}\left[\left(1+\bar{n}_{t+1}\right) \bar{\pi}_{1, t+1}-\left(1+\bar{n}_{t}\right)\right], \\
g_{22} & =\left(1-\bar{w}_{t}\right) \bar{\pi}_{2, t}\left[\left(1-\bar{n}_{t+1}\right) \bar{\pi}_{2, t+1}-\left(1-\bar{n}_{t}\right)\right]
\end{aligned}
$$

and $\bar{\pi}_{j, t}(j=1,2)$ are defined by (2.4). The difference of population proportions $n_{t}$ evolves according to

$$
n_{t+1}=\tanh \left[\frac{\beta}{2}\left(\left(\Phi_{1, t}-\Phi_{2, t}\right)-\left(C_{1}-C_{2}\right)\right)\right],
$$

where the fitness functions are defined as

$$
\Phi_{j, t+1}=\bar{\pi}_{j, t}\left[r+\left(\rho_{t+1}-r\right) \bar{\pi}_{j, t}\right]+\gamma \Phi_{j, t},
$$

and $C_{j} \geq 0$ measure the cost of the strategy for $j=1,2$.

3.3. Wealth distribution and profitability of trading strategies. The average wealth distribution among two types of agents (following different trading strategies) is now characterized by $\bar{w}_{t}$, the difference of the average wealth proportions. Over a certain time period, if $\bar{w}_{t}$ stays above (below) the initial value $\bar{w}_{o}$ and increases (decreases) significantly as $t$ increases, then, on average, type 1 agents accumulate more (less) wealth than type 2 agents, and one may say type 1 trading strategy is more (less) profitable than type 2 trading strategy. Otherwise, if the difference is not significantly different from $\bar{w}_{o}$, then there is no evidence that on average either trading strategy is more profitable than the other.

3.4. Population distribution and herd behavior. The distribution on populations using different types of trading strategies is now characterized by the difference of the population proportions $n_{t}$. At time period $t$, if $n_{t}$ is positive (negative), then this indicates that there are more (less) agents using type 1 trading strategy than type 2 trading strategy. Moreover, if $n_{t}$ is significantly different from zero, then this could be taken as an indication of herd behavior. This is, in particular, frequently observed to be the case when the switching intensity $\beta>0$ is high.

When there is evidence on the profitability of type 1 (type 2) trading strategy and a clear indication on herd behavior using type 1 (type 2) trading strategy over the time period, we say type 1 (type 2) trading strategy dominates the market. 
3.5. A Quasi-Homogeneous Model. As a special case of the adaptive model with two types of agents, consider the case, termed quasi-homogeneous model, where both types of agents use exactly the same trading strategies except that they use different memory lengths.

The trading strategies for both types of agents can be unified by writing

$$
E_{i, t}\left(\rho_{t+1}\right)=r+\delta_{i}+d_{i} \bar{\rho}_{i, t}, \quad \bar{\rho}_{i, t}=\frac{1}{L_{i}} \sum_{k=1}^{L_{i}} \rho_{t-k},
$$

for $i=1,2$, where $L_{i} \geq 1$ is integer, $r(>0), \delta_{i}(>0)$ and $d_{i} \in \mathbb{R}$ are constants. For the quasi-homogeneous model, it is further assumed that $\delta_{1}=\delta_{2}=\delta, d_{1}=d_{2}=d$ but $1 \leq L_{1} \leq L_{2}$.

In the following discussion, assume that the conditional variances of agents are given by a constant $\sigma^{2}$. It is convenience to standardize both the risk premium $\delta$ and extrapolation rate $d$ according to:

$$
\bar{\delta}=\frac{\delta}{\sigma^{2}}, \quad \bar{d}=\frac{d}{\sigma^{2}} .
$$

Correspondingly, the optimal demand of type $j$ agents in terms of the wealth proportion invested in the risky asset is given by

$$
\pi_{j, t}=\bar{\delta}_{j}+\bar{d}_{j} \bar{\rho}_{j, t} .
$$

It is also assumed that the dividend yield process has the form

$$
\alpha_{t}=\alpha_{o}+q \mathcal{N}(0,1),
$$

where $\mathcal{N}(0,1)$ is the standard normal distribution. ${ }^{13}$

Because of the highly nonlinear nature of the adaptive model theoretical analysis (even of the steady states) seems intractable and thus the model is analyzed numerically. However, the results on the non-adaptive model established in Chiarella and $\mathrm{He}$ (2001) underly the dynamics of the adaptive model established here. In the presence of heterogeneous agents, the non-adaptive model can have multiple steady states, and the convergence of such steady states follows an optimal selection principle-the return and wealth proportions tend to the steady state which has relatively high return. More importantly, heterogeneity can generate instability which, under the stochastic noise processes, results in switching of the return among different states, such as steadystates, periodic and aperiodic cycles from time to time. One would expect the adaptive model to display even richer dynamics.

3.5.1. Existence of steady-state returns. If $\alpha_{t}=\alpha_{o}$ is a constant, the system (3.2)(3.6) becomes a deterministic dynamical system. The return time series generated by the adaptive model is the outcome of the interaction of this deterministic dynamical system with external noise processes (here the dividend yield process). A first step to understanding the possible dynamical behavior of the noise perturbed dynamical system is an understanding of the underlying dynamics of the deterministic systems, such as existence of steady-states, their stability and bifurcation.

When $\alpha_{t}=\alpha_{o}$ is a constant, in terms of steady-state of return and wealth proportions, it is easy to see that the quasi-homogeneous model has the same steady-state as

\footnotetext{
${ }^{13}$ The normal distribution has been chosen for convenience, it has the disadvantage that the dividend yield $\alpha_{t}$ could be negative. However for the parameters used in the simulations this probability is extremely low so the distribution is truncated at 0 .
} 
the homogeneous model (in which $L_{1}=L_{2}$ ). The existence of such steady states is studied in Chiarella and He (2001) and the results may be summarized as follows:

- The steady-state of the wealth proportions stays at the initial level, while the steady-state of the return depends on the extrapolation rate $\bar{d}$.

- There is a unique steady-state return when $\bar{d}=0$. In other words, when agents are fundamentalists, there is a unique steady-state return which, for convenience of discussion, is called the fundamental steady-state return. Moreover, high risk premia $\delta$ correspond to high levels of the steady-state return.

- There exist two steady-state returns when $\bar{d}<0$, that is when agents are contrarians. One of the steady-state returns is negative while the other is positive, the positive steady-state return is called the contrarian steady-state return. More importantly, with the same risk premium, when agents act as contrarians, the contrarian steady-state return is pushed below the fundamental steady-state return.

- There exist two steady-state returns when $\bar{d}(>0)$ is small. That is, when agents are momentum traders and they extrapolate weakly, the return has two positive fixed steady states. However, when $\bar{d}$ is close zero, only one of the steady states is bounded and this steady-state return is called the momentum steady-state return. Furthermore, given the same risk premium, compared to the fundamental equilibrium, a weakly homogeneous momentum trading strategy (i.e. $\bar{d}>0$ small) leads to a higher level of steady-state return.

An aim of the following analysis is to determine to what extent the adaptive model for two types of agents reflects these characteristics.

3.5.2. Parameters and initial value selection. Using data for the United States during the 1926-94 period, as reported by Ibbotson Associates, the annual risk-free interest rate, $r=3.7 \%$, corresponds to the average rate during that period. The initial history of rates of return on the stock consists of a distribution with a mean of $12.2 \%$ and a standard derivation of $20.4 \%$. A mean dividend yield of $\alpha_{o}=4.7 \%$ corresponds to the historical average yield on the $S \& P 500$. The initial share price is $p_{o}=\$ 10.00$.

The analysis in the following sections selects the annual risk-free rate $r$, standard derivation $\sigma$ and the mean dividend yield $\alpha_{o}$ as indicated above. For the simulations, the time period between each trade is one day and simulations are conducted over 20 years. Parameters and initial values are selected as follows, unless stated otherwise,

$$
\bar{\delta}=0.6, \beta=0.5, \gamma=0.5, C_{1}=C_{2}=0
$$

and

$$
\bar{w}_{o}=0, n_{o}=0, \Phi_{1, o}=\Phi_{2,0}=0.5, p_{o}=\$ 10 .
$$

Furthermore, annual rates of risk-free rate and returns of the risky asset are used in the fitness functions $\Phi_{j, t}$ for $j=1,2$.

\section{Wealth Dynamics of Momentum Trading Strategies}

This section considers the quasi-homogeneous model with $d_{1}=d_{2}=d>0$ and $1 \leq L_{1}<L_{2}$, that is both types of agents follow the same momentum trading strategy except for having different memory lengths. The simulations address the question as to which type of agent dominates the market over the time. 
As discussed in section 2, some empirical studies seem to support a view that momentum strategies are profitable over short time intervals, but not over long time intervals. The following discussion examines different combination of $\left(L_{1}, L_{2}\right)$ and analyzes the effect of lag length on the wealth dynamics ${ }^{14}$. The results indicate in general that, the strategy with short memory length dominates the market by accumulating more wealth and attracting more of the trading population. The adaptive model outlined in this paper is thus capable of characterizing some broad features found in empirical studies.

4.1. Case: $\left(L_{1}, L_{2}\right)=(3,5)$. The following subsection considers first the dynamics of the underlying deterministic system, that is, when $q=0$ in (3.8). The impact of the noise processes on the dynamics is then considered in the subsequent subsection.

4.1.1. No-noise Case. For $\bar{d}=0.5$, initial population proportion $n_{o}=0$ and any initial wealth proportion $\bar{w}_{o}$, numerical simulations show that

$$
\rho_{t} \rightarrow \rho^{*}=15.45 \% \quad \text { (annualized), } \quad \bar{w}_{t} \rightarrow \bar{w}_{o}, \quad n_{t} \rightarrow 0 .
$$

By changing various parameters and initial values, the following results on the momentum trading strategies from the quasi-homogeneous model have been obtained.

(i) Risk premium and over-pricing.

It is found that, ceteris paribus, for $\bar{\delta}\left(=\delta / \sigma^{2}\right)=0.35, \rho_{t} \rightarrow \rho^{*}=10.94 \%$, while for $\bar{\delta}=0.53, \rho^{*}=15.45 \%$. In general, a high level of risk-adjusted premium leads to a high return, and a high price as well. In fact, for the given parameters, there exists $\bar{\delta}_{o} \in(0.69,0.7)$, so called bifurcation value ${ }^{15}$, such that the returns converge to fixed values for $\bar{\delta}<\bar{\delta}_{o}$ and diverge for $\bar{\delta}>\delta_{o}$, leading to price explosion.

(ii) Over-extrapolation and overshooting.

Momentum traders form expectations of future prices by extrapolating trends. However, when the prices or returns are over-extrapolated, stocks are overpriced, and as a result, overshooting takes place. Based on the parameters selected, simulations indicate that there exists $d_{o} \in(0.573,0.574)$ such that, ceteris paribus, returns converge to fixed values for $\bar{d}<d_{o}$ and diverge for $\bar{d}>d_{o}$, leading prices to exhibit overshooting.

(iii) No noise, no effects on population and wealth distribution and no herding behavior.

For either fixed $n_{o} \neq 0$ and a range of $\bar{w}_{o}$ (say $n_{o}=-0.3$ and $\bar{w}_{o} \in(-0.5,0.3)$ ), or fixed $\bar{w}_{o} \neq 0$ and a range of $n_{o}$ (say, $\bar{w}_{o}=-0.3$ and $n_{o} \in(-1,0.3)$ ), simulations show that

$$
\rho_{t} \rightarrow \rho^{*}=15.45 \% \quad \text { (annual), } \quad \bar{w}_{t} \rightarrow \bar{w}_{o}+\epsilon, \quad n_{t} \rightarrow 0
$$

with $\epsilon \approx 10^{-6}$. Also, the switching intensity parameter $\beta$ has almost no effect on the results (as long as the returns series converge to constants). This implies that, without the noise from the dividend yield process, in terms of profitability,

\footnotetext{
${ }^{14}$ The selection of various combinations of lag lengths is arbitrary. However more extensive simulations (not reported) indicate some robustness of the results presented in this paper.

${ }^{15}$ As in Brock and Hommes (1998), the dynamics of the system through various types of bifurcation can be analyzed and are of interest. However, in this paper, we focus on the dynamics of the stochastic system when the return process of the underlying deterministic system is stable.
} 
type 1 trading strategy is slightly better than type 2, but not significantly. In addition, the populations of agents using different strategies become evenly distributed. In other words, no one of the momentum trading strategies dominates the market, even though both wealth and population are not evenly distributed initially. Therefore, when there is no noise from the dividend yield process and the returns converge to constants, the average wealth proportions, as expected, stay at their initial level, while the average population proportions are evenly distributed, and there is no herd behavior.

4.1.2. Effect of Noise. Select the annualized standard derivation of the noisy dividend yield process, $q=0.03=3 \%$. When adding a noisy dividend process to the adaptive system, the general features of the corresponding deterministic system (without the noise), such as the results (i)-(ii) above, still hold. However, it has a significant impact on the dynamics of the system, such as wealth and population distributions, autocorrelation of returns, volatility of returns and prices etc., as indicated below. In particular, the dynamics of the model is greatly affected by agents' behavior, which is measured by their extrapolation rate, $\bar{d}$, and switching intensity, $\beta$. The following discussion is focused on the dynamics of the system for various combinations of these two parameters $\bar{d}$ and $\beta$.

The following simulation results are based on the parameters selected above, unless otherwise indicated.

- Wealth distribution.

Wealth distribution is largely influenced by agents' extrapolation and strategy switching activity. Simulations show that, in general, a strong extrapolation leads type 1 trading strategy (with lag 3 ) to accumulate more wealth than type 2 trading strategy does. In other words, type 1 trading strategy(with lag of 3) is more profitable than type 2 (with lag of 5) under the noisy dividend process. Furthermore, as the switching intensity $\beta$ increases, the profitability of type 1 trading strategy is improved significantly. This result is unexpected and interesting, and it is optimal in the sense that the overall outcome is independent of the initial wealth and population distributions.

- Effect of the initial wealth distribution.

When the wealth and population are evenly distributed across the two types of strategies initially (i.e. $\bar{w}_{o}=0, n_{o}=0$ ), on average, type 1 strategy accumulates more wealth (about 5\% to 6\%) than type 2 strategy over the whole period, as indicated by the time series plot for the average wealth proportion difference $\left(\bar{w}_{t}\right)$ in Figure 4.1. Also, as the extrapolations rate increases (i.e. as $\bar{d}$ increases), type 1 strategy accumulates more wealth than type 2 strategy (say, about $2 \%$ to $3 \%$ more for $\bar{d}=0.5$, compared to 5-6\% more for $\bar{d}=0.53$ ). This suggests that, when both types of strategies start with the same level of wealth and have the same number of traders, type 1 trading strategy is more profitable under the noisy dividend process. This result still holds when the initial wealth is not so evenly distributed. However, on average, when type 1 strategy starts with more wealth than type 2 (say $\bar{w}_{o}=0.2$, that is type 1 has $20 \%$ more initial wealth than type 2 on average), the prices can be pushed immediately to very high levels so that any further trend chasing from type 1 strategy can cause price to overshoot, leading an explosion of price. 


\section{- Effect of the initial population distribution}

For a fixed initial wealth proportion $\bar{w}_{o}$ (say $\bar{w}_{o}=0$ ) and a range of $n_{o}$ (say, $n_{o} \in(-1,0.5)$ ), $\bar{w}_{t}$ increases in $t$. But for large $n_{o}$ (say $n_{o}=0.6$ ), the prices are pushed to explosion. This indicates that type 1 strategy accumulates more wealth over the period, even when the population of type 2 agents is high initially. However, an initial over concentration of type 1 agents can lead to overshooting of price.

- Herding behavior.

Herding behavior is measured by the population proportion difference $n_{t}$ and the switching intensity parameter $\beta$. For $\beta=0$, there is no switching between the two trading strategies. However, when agents are allowed to switch (i.e., $\beta>0)$, as indicated by the time series plot for the population $\left(n_{t}\right)$ in Figure 4.1, agents switch between the two strategies frequently. In general, because of the profitability of type 1 strategy, more agents switch from type 2 to type 1 , as indicated by the mean and standard deviation of the population $n_{t}$ in Table A.2.1. Also, as the switching intensity $\beta$ increases, simulations (not reported here) show that the frequency of such switching increases too. Furthermore, as $\beta$ increases, both prices and returns become more volatile, as indicated by the time series plots on returns $\left(\rho_{t}\right)$ and prices $\left(p_{t}\right)$ in Figure 4.1.

- Excess volatility and volatility clustering.

As indicated by the time series plot of returns $\rho_{t}$ in Figure 4.1, adding the noisy dividend process causes an otherwise stable return series to fluctuate. This fact itself is not unexpected. What is of interest is the contrast between the simply normally distributed dividend process that is input to the system and the return process that is the output of the system. With the increase of either the standard derivation of the noise process $q$, or agents extrapolation rate $\bar{d}$, or switching intensity $\beta$, both returns and prices become more volatile. Moreover, volatility clustering is also observed.

- Autocorrelation.

Significant positive autocorrelation (AC) for lags 1 and 2, negative for lags 3 to 8, positive for lags 9-14, are founded, as indicated by Table A.2.4. However, as lag length increases, the ACs become less significant.

- Overshooting.

Related simulations (not reported here) indicate that either strong extrapolation (corresponding to high $\bar{d}$ ), or high volatility of the dividend yield process $(q)$, or high switching density $(\beta)$ can cause price to overshoot and lead to price explosion. Numerical simulations also show that, to avoid price overshooting, a minimum level of risk premium $(\bar{\delta})$ is required.

4.2. Other Lag Length Combinations. This section addresses how the above results are affected by different lag length combinations.

- $\left(L_{1}, L_{2}\right)=(3,7)$ : The general dynamic features are observed to be similar to the case when $\left(L_{1}, L_{2}\right)=(3,5)$, except for the following differences:

- The underlying deterministic system is stable over a wider range of extrapolation rates $\bar{d} \in\left[0, d^{*}\right) ; d^{*} \approx 0.57$ for $L_{2}=5$ and $d^{*} \approx 0.773$ for $L_{2}=7$.

- The trading strategy with short lag dominates the market. Similar impacts of initial wealth and population distributions, the switching intensity, and 

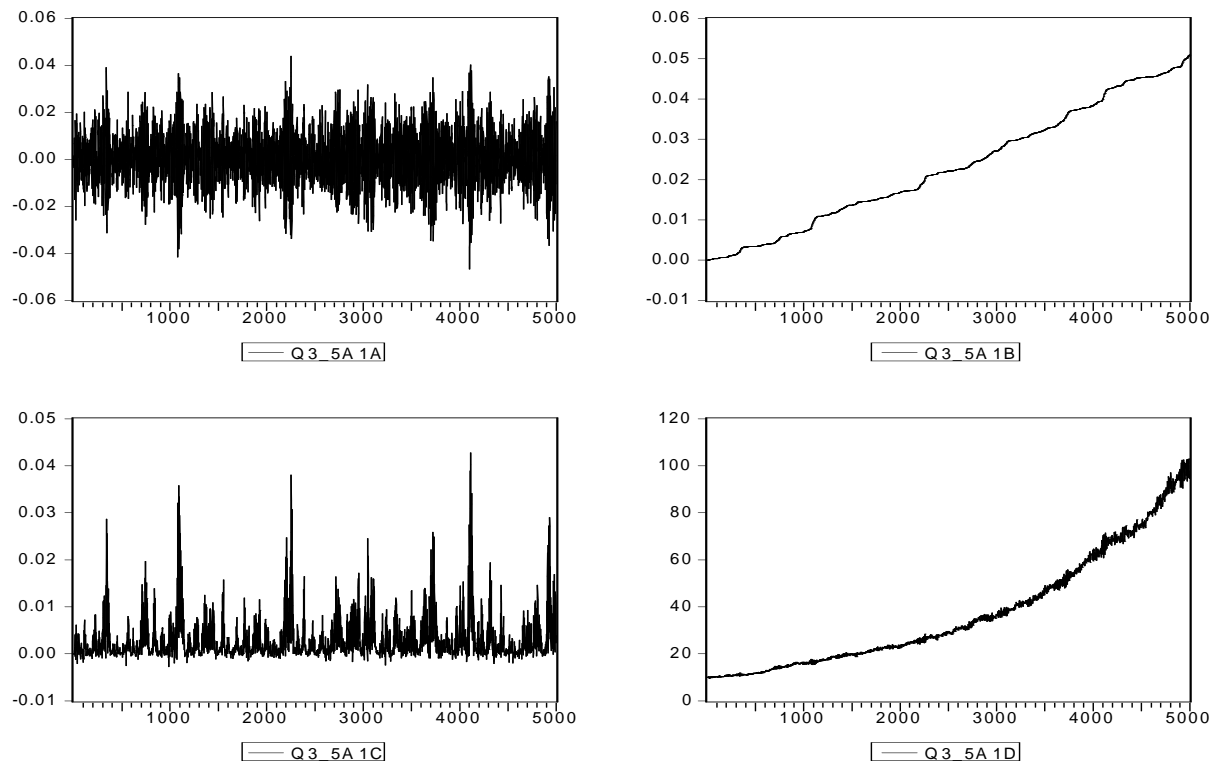

FIGURE 4.1. Time series plots for returns (top left), wealth (top right) and population (bottom left) distributions, and prices (bottom right) when the same momentum trading strategies with different lags $\left(L_{1}, L_{2}\right)=(3,5)$ are used. Here, $\bar{\delta}=0.6, \bar{d}=0.53$ and $q=0.03$.

the standard deviation of the noisy process on the returns and wealth dynamics are also observed over a wider range of the parameters and initial values. However, compared with the previous case, for the same set of parameters and initial values, both the profitability and herd behavior increase, as indicated by the time series plots for wealth and population in Figure A.2.1 and the corresponding statistical results in Table A.2.1.

- ACs are significantly positive for lags 1 and 2, either positive or negative for lag 3, but not significantly, negative for lags 4 to 9 , positive for lags 10 to 15 , as indicated in Table A.2.4 .

- $\left(L_{1}, L_{2}\right)=(10,14)$ : Compared with the simulations of the previous two cases, the following differences have been observed.

- The upper bound $d^{*}$ for returns of the underlying deterministic system to be stable increases to $d^{*} \approx 1.57$.

- By adding the noisy dividend yield process, the trading strategy with memory length 10 accumulates more wealth than the one with lag length 14. However, in contract to the previous cases, for the same set of parameters and initial values, the profitability and herd behavior of the strategy of lag 10 compared to the one with lag 14 is much less significant (at about $0.1 \%$ to $0.2 \%$ ), as indicated by the time series plots in Figure A.2.2 and the corresponding statistical results in Table A.2.2; although an increase of extrapolation improves the profitability of the strategy with lag 10, as 
indicated by the time series plots in Figure 4.2 and the corresponding statistics in Table A.2.2 for $\bar{d}=1.2$.

- Indicated by Table A.2.4, ACs oscillate and become less significant when agents extrapolate weakly (say, for $\bar{d}=0.5$ ), but become more significant when agents extrapolate strongly (say, $\bar{d}=1.1$ ).

- There is less herding behavior than in the previous cases. This is partially because of the less significant profitability of one strategy with lag 10 over the other with lag 14.
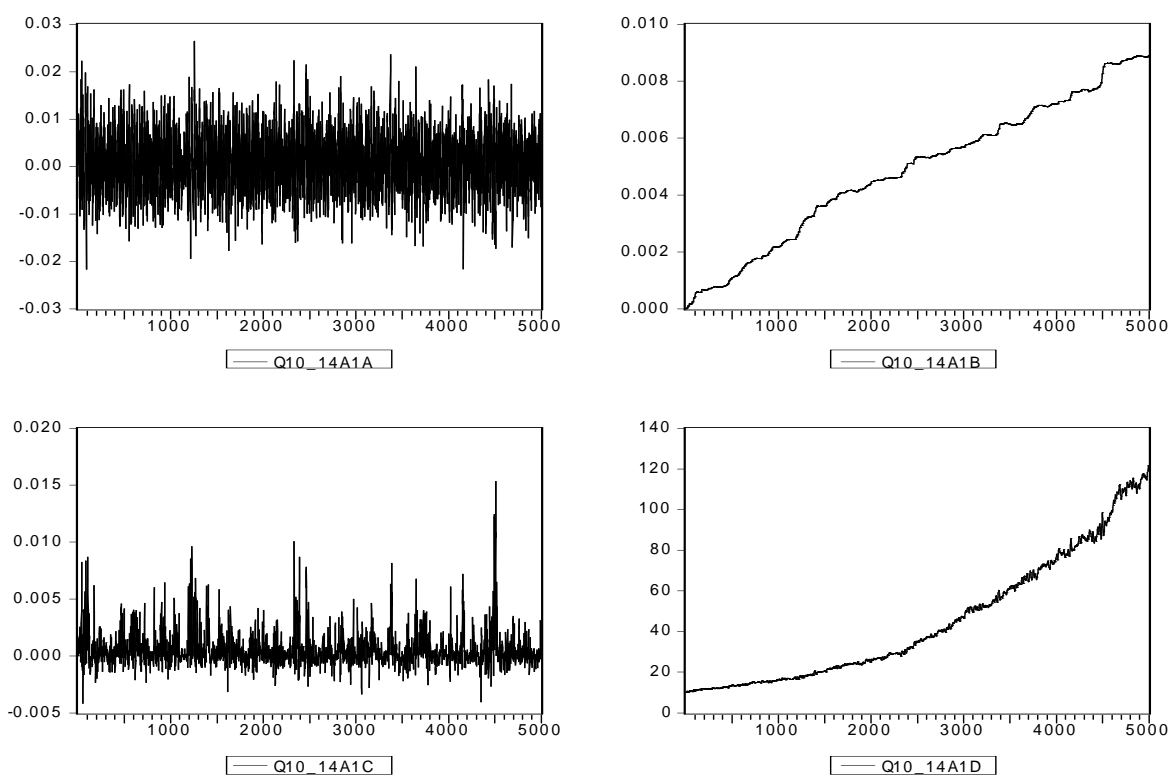

FIGURE 4.2. Time series plots for wealth and population distributions, returns and prices when the same momentum trading strategies with different lags $\left(L_{1}, L_{2}\right)=(10,14)$ are used. Here, $\bar{\delta}=0.6, \bar{d}=1.2$ and $q=0.03$.

- $\left(L_{1}, L_{2}\right)=(10,26)$ :

- The upper bound $d^{*}$ for returns of the underlying deterministic system to be stable increases to $d^{*} \in(2.2,2.3)$.

- By adding the noisy dividend yield process, with the same parameters and initial values, profitability of type 1 trading strategy (with lag length 10) becomes questionable, as indicated by the time series plots in Figures A.2.3 and 4.3 and the corresponding statistics in Table A.2.3.

- As demonstrated by Table A.2.4, the ACs have less patterns and do not die out as lags increase. 

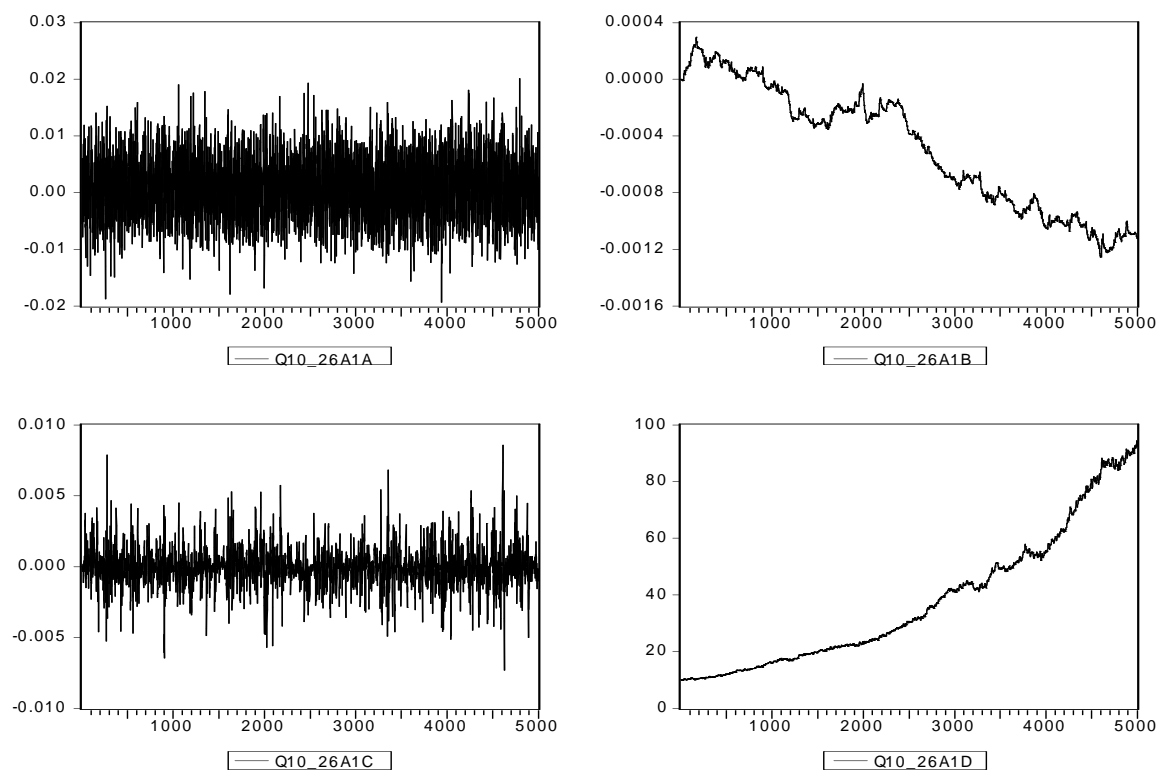

FIGURE 4.3. Time series plots for wealth and population distributions, returns and prices when the same momentum trading strategies with different lags $\left(L_{1}, L_{2}\right)=(10,26)$ are used. Here, $\bar{\delta}=0.6, \bar{d}=$ $1.2, q=0.03$ and $\bar{w}_{o}=0.6$.

4.2.1. Summary. In summarizing, we obtain the following results when both types of agents follow the same momentum trading strategy, but with different memory lengths.

- Without the noisy dividend yield process, an increase in lag length from either one of the trading strategies stabilizes the return series of the underlying deterministic system, and enlarges the range of the extrapolation coefficient for which the market does not explode. However, for the same set of parameters, the profitability of the trading strategies and herd behavior become less significant.

- Adding the noisy dividend process in general improves the profitability of the trading strategies with short lag length $L_{1}$ (say, $\left(L_{1}, L_{2}\right)=(3,5)$ and $(3,7)$ ). However, such profitability becomes less significant when the short lag length $L_{1}$ increase, and may even disappear (say $\left(L_{1}, L_{2}\right)=(10,14)$ and $(10,26)$ ).

- When trading strategies become profitable, agents tend to adopt a herd behavior - more agents switch to the more profitable strategy over the time period. However, over concentration (in terms of the initial average wealth, population proportion), or over extrapolation (in terms of high extrapolation rates and improper risk premium levels) can cause overshooting of price and push prices to explosion, leading to a market crash. 
- Momentum trading strategies can push the prices to a very high level and lead the returns to be more volatile, exhibiting volatility clustering.

- ACs follow certain patterns when one of the trading strategies becomes profitable and die out as lags increase. However, such patterns become insignificant when the profitability of the trading strategy becomes less significant, and may even disappear.

- Price levels are more determined by the risk premium levels rather than other parameters (say extrapolation rate and switching intensity).

\section{Wealth Dynamics of Contrarian Trading Strategies}

This section considers the quasi-homogeneous model with $d_{1}=d_{2}=d<0$ and $1 \leq L_{1}<L_{2}$, that is both types of agents follow the same contrarian trading strategy except for having different memory lengths. As discussed in Section 3, some empirical studies suggest that contrarian trading strategies are more profitable over long periods. The results in this section provide some consistency with this view and show that the adaptive model presented in this paper is capable of characterizing some features found in empirical studies. Furthermore, similar to the previous section, wealth and population distributions, statistical properties of returns (such as volatility clustering, autocorrelations), and herd behavior are discussed.

5.1. Case: $\left(L_{1}, L_{2}\right)=(3,5)$. With the selection of the parameters and initial values in (3.8)-(3.9), consider first in the next subsection the dynamics of the underlying deterministic system, that is, when $q=0$. The impact of the noisy dividend yield process on the dynamics is then studied in the subsequent subsection.

5.1.1. No-noise Case. Let $q=0$. For $\bar{d}=-0.4$, initial difference of population proportions $n_{o}=0$ and any initial wealth proportion $\bar{w}_{o}$, it is found that

$$
\rho_{t} \rightarrow \rho^{*}=15.45 \% \quad \text { (annual), } \quad \bar{w}_{t} \rightarrow \bar{w}_{o}, \quad n_{t} \rightarrow 0 .
$$

By changing parameters and initial values, the following results are obtained.

- Risk premium and over-pricing

It is found that, ceteris paribus, for $\bar{\delta}\left(=\delta / \sigma^{2}\right)=0.4, \rho_{t} \rightarrow \rho^{*}=11.52 \%$, while for $\bar{\delta}=0.6, \rho^{*}=15.45 \%$. In general, a high level of risk-adjusted premium leads to a high return and a high price correspondingly. In fact, for the given parameters, there exists $\bar{\delta}_{o} \in(0.6,0.7)$, a so called bifurcation value, such that the returns converge to fixed values for $\bar{\delta}<\bar{\delta}_{o}$ and diverge for $\bar{\delta}>\delta_{o}$, leading prices to explode.

- Over-reaction and price shooting

Based on the parameters selected, there exists $d_{o} \in(-0.53,-0.52)$ such that, ceteris paribus, returns converge to fixed values for $(0>) \bar{d}>d_{o}$ and diverge for $\bar{d}<d_{o}$, leading prices to overshoot. Like the momentum trading strategies, over-extrapolation from contrarian trading strategies also causes overshooting of prices.

- Wealth distribution

Unlike the case of the momentum trading strategies, wealth distributions of the deterministic system are affected differently by the extrapolation rate $\bar{d}$, switching intensity, initial wealth and population distributions. 
- In general, as $\bar{d}(<0$ decreases and is near the bifurcation value, the profitability of trading strategy with long lag $(L=5)$ is improved significantly, say from $5 \%$ for $\bar{d}=-0.454$, to $25 \%$ for $\bar{d}=-0.48$, and to $50 \%$ for $\bar{d}=-0.5$. However, for fixed $\bar{d}<0$, say $\bar{d}=-0.5$, as $\beta$ increases, the profitability of trading strategy 2 becomes less significant, say from $45 \%$ for $\beta=0.1$ to $20 \%$ for $\beta=2$. This is different from the case of momentum trading strategies.

- For fixed $n_{o} \neq 0$ and a range of $\bar{w}_{o}$ (say, $n_{o}=0.3$ and $\bar{w}_{o} \in(-0.5,0.5)$ ),

$$
\rho_{t} \rightarrow \rho^{*}=15.45 \% \quad \text { (annual), } \quad \bar{w}_{t} \rightarrow \bar{w}_{o}-\epsilon, \quad n_{t} \rightarrow 0
$$

with $\epsilon \approx 10^{-6}$. This implies that agents' wealth are distributed according to their initial wealth distribution, although populations are not evenly distributed initially.

- For fixed $\bar{w}_{o}<0$ (say, $\bar{w}_{o}=-0.3$ ) and $n_{o} \in[-1,1]$, type 2 strategy accumulated more wealth than type 1 strategy over a very short period, but the difference is not significant (about 1\%). In other words, when the initial average wealth for type 2 strategy is more than average wealth for type 1 strategy, no one of the contrarian trading strategies can make significant profit over the other, no matter how the initial populations are distributed.

- For fixed $\bar{w}_{o}>0$ (say, $\bar{w}_{o}=0.3$ ) and $n_{o} \in[-1,1]$, type 2 strategy accumulates more wealth than type 1 strategy over a very short period, and the difference becomes more significant (up to 37\%) as more agents use type 2 trading strategy initially. This implies that, when the initial average wealth of type 1 strategy is higher than the one of type 2 strategy, contrarian strategies with long memory length $\left(L_{2}=5\right)$ are able to accumulate more wealth over a very short period than the same strategy but with short memory length $\left(L_{1}=3\right)$. In addition, the profitability becomes more significant when there are more agents using the strategy with long memory length initially. This is different from the case when agents use momentum strategies.

\section{- Herding behavior}

The dynamics display no significant differences for different switching intensity parameter $\beta$ when the returns process for the underlying deterministic system is stable. However, as $\bar{d}$ near the bifurcation value, herd behavior is also observed.

5.1.2. Effect of Noise. Let the annualized standard derivation of the noisy dividend yield process be $q=3 \%$. The following results are based on the parameters selected above, unless the difference is indicated.

- Wealth distribution

- Effect of the initial wealth distribution - When the wealth and population are evenly distributed among the two types of trading strategies initially (i.e. $\bar{w}_{o}=0, n_{o}=0$ ), type 2 strategy accumulates more average wealth (about 5\% to 7\%) more than type 2 strategy over the whole period, as indicated in Figure 5.1 for the time series plots of wealth and population. Also, as extrapolations increase (i.e. as $\bar{d}$ decreases), such extrapolations 
help type 2 strategy to accumulate more wealth than type 1 strategy (say, about $5 \%$ for $\bar{d}=-0.45$, and about $45 \%$ for $\bar{d}=-0.5$ ). This suggests that, when both types of strategies start with the same level of wealth and have equal number of traders, the strategy with long memory length $\left(L_{2}=5\right)$ accumulate more wealthy than one with short memory length $\left(L_{1}=3\right)$. In other words, type 2 strategy benefits significantly from the noisy dividend yield process. This result still holds when the initial wealth is not so evenly distributed (say, $\bar{w}_{o} \in(-0.6,0.3)$ for $\left.\bar{d}=-0.5\right)$.
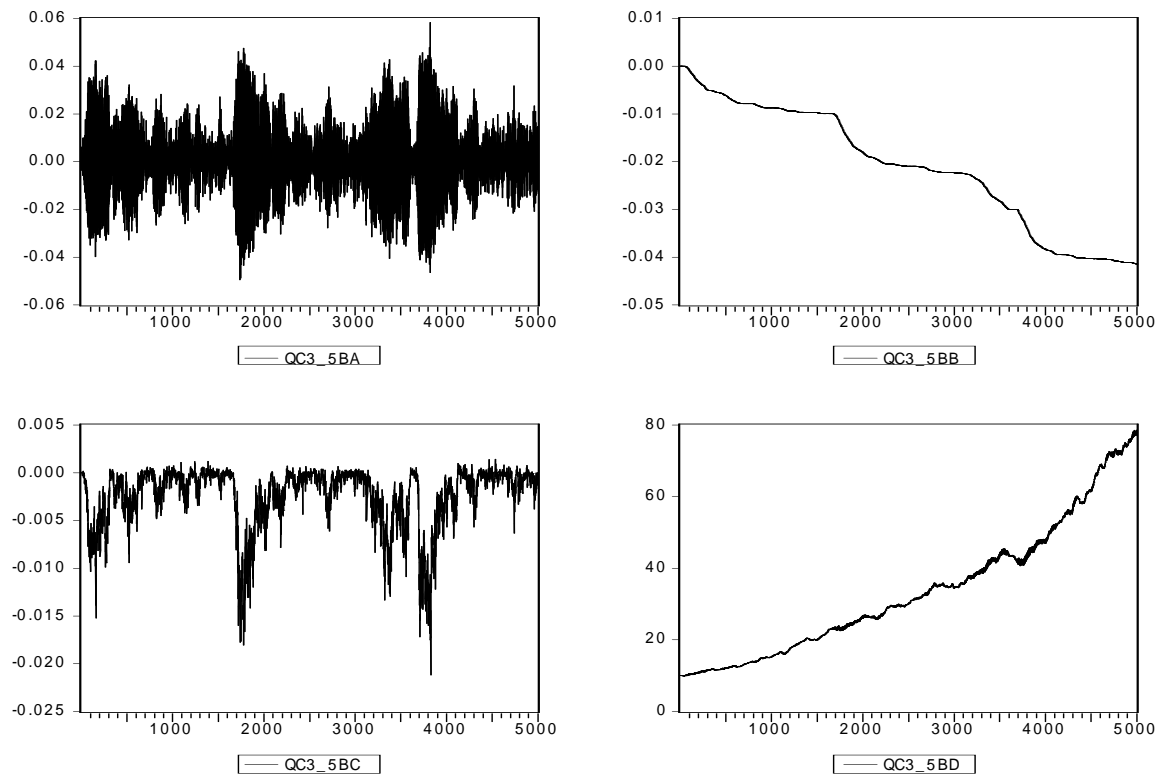

FIGURE 5.1. Time series plots for wealth and population distributions, returns and prices when the same contrarian trading strategies with different lags $\left(L_{1}, L_{2}\right)=(3,5)$ are used. Here, $\bar{\delta}=0.6, \bar{d}=$ -0.45 and $q=0.03$.

- Effect of the initial population distribution - Similar to the case without noise, the wealth distribution is affected differently as a function of different initial wealth levels. For fixed $\bar{w}_{o}<0$ and a range of $n_{o}$ (say, $\bar{w}_{o}=-0.3$ and $\left.n_{o} \in(-0.8,0.65)\right)$, the profitability of trading strategy 2 does not change much for different $n_{o}$. However, for fixed $\bar{w}_{o}>0$ and a range of $n_{o}$ (say, $\bar{w}_{o}=0.3$ and $n_{o} \in(-0.9,0.9)$ ), the profitability of trading strategy 2 increases significantly as more and more agents use trading strategy 2. Price overshooting is possible when the populations are over concentrated in use of one of the trading strategies.

\section{- Herding behavior}

Herding behavior is also observed for changing values of the parameter $\beta$. 
Given the profitability of the trading strategy over the long memory span, more agents tend to switch to this more profitable strategy, as indicated by the time series plot of population in Figure 5.1 and the corresponding statistics in Table A.2.5. Furthermore, as $\beta$ increases, both prices and returns become more volatile, leading to excess volatility.

- Excess volatility and volatility clustering

The addition of a noisy dividend yield process cause an otherwise stable return series to be exhibit fluctuations. Similar to the case of using momentum trading strategies, an increase of either the standard deviation of the dividend yield noisy process $q$, agents extrapolation $\bar{d}$, leads both returns and prices to be more volatile. Moreover, volatility clustering is also observed, as illustrated by the time series plot of the returns in Figure 5.1 and the corresponding statistics in Table A.2.5.

- Autocorrelation

ACs are significantly negative for odd lags and positive for even lags for all lags, as indicated in Table A.2.6.

- Overshooting - Similar to the momentum trading strategies discussed in Section 4 , the noisy dividend yield process has a significant impact on prices. An increase of $q$ can push prices to significantly high levels. This can also result from either strong extrapolation (corresponding to low $\bar{d}$ ), or high risk premia $\bar{\delta}$, or high switching density $\beta$ and causes prices to explode.

\subsection{Other Cases.}

- $\left(L_{1}, L_{2}\right)=(3,7)$ : The general dynamic features are similar to the above case when $\left(L_{1}, L_{2}\right)=(3,5)$, except for the differences indicated below.

- The underlying deterministic system is stable over a wider range of extrapolation rates $\bar{d} \in\left(d^{*}, 0\right]$ with $d^{*} \in(-0.65,-0.6)$ for $L_{2}=7$ in contrast with $d^{*} \in(-0.53,-0.52)$ for $L_{2}=5$.

- Similar to the previous case, the trading strategy with the longer lag $L_{2}=$ 7 dominates the market,in particular, when $\bar{d}$ is near the bifurcation value. However, for the same set of parameters, compared with the case of $L_{2}=$ 5 , the profitability is reduced slightly. On the other hand, agents can extrapolate over a wide range (of the parameter $\bar{d}$ ). Similar impacts of initial wealth and population distributions, the switching intensity, and the standard derivation of the noisy dividend process on the dynamics can be observed over a wider range of the parameters.

- $\left(L_{1}, L_{2}\right)=(10,14)$ :

- The lower bound $d^{*}$ for dynamic process for returns of the underlying deterministic system to be stable decreases to $d^{*} \in(-1.6,-1.5)$. By adding the noisy dividend yield process, with the same parameters and initial values, the profitability of type 1 trading strategy (with lag length 14) becomes questionable, as indicated by the time series plots of the wealth in Figure 5.2 and the corresponding statistics in Table A.2.5.

- The patterns of the ACs are maintained, but they become less significant (for the same parameter $\bar{d}=-0.45$ ), as shown in Table A.2.6.

- Compared with the previous cases, there is less herd behavior, as illustrated by the time series plot for the population in Figure 5.2 and the corresponding statics in Table A.2.5. This is partially because of the less 
significant (even no) profitability of the strategy with lag 14 over the other with lag 10.
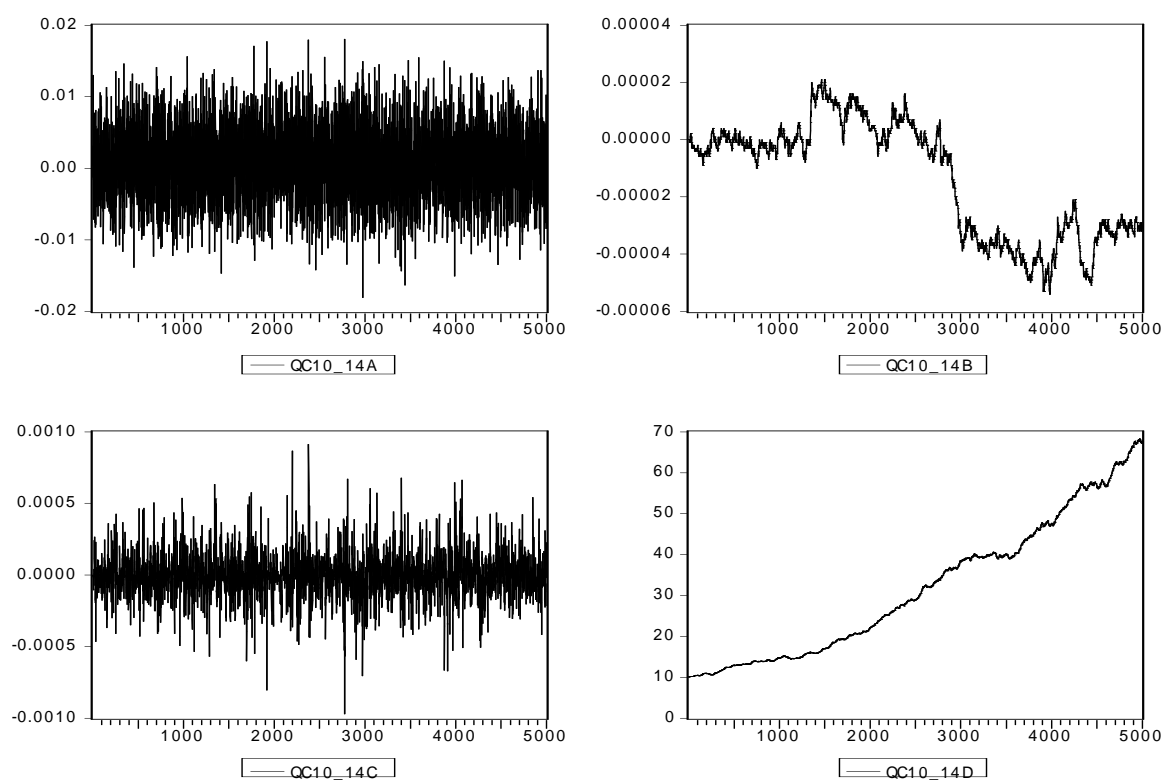

FIGURE 5.2. Time series plots for wealth and population distributions, returns and prices when the same contrarian trading strategies with different lags $\left(L_{1}, L_{2}\right)=(10,14)$ are used. Here, $\bar{\delta}=0.6, \bar{d}=$ -0.45 and $q=0.03$.

- $\left(L_{1}, L_{2}\right)=(10,26)$ : The following differences have been observed.

- In this case the lower bound $d^{*}$ (on $\bar{d}$ ) such that the dynamic process for returns of the underlying deterministic system be stable decreases to $d^{*} \in(-2.2,-2.1)$. By adding the noisy dividend yield process, the trading strategy with memory length 26 accumulates more wealth than the one with lag length 10, as shown in Figure 5.3 and Table A.2.5. However, comparing with the previous cases $\left(L_{1}, L_{2}\right)=(3,5),(3,7)$, the profitability of the strategy of lag 26 over the one with lag 10 is much less significant (at about $0.01 \%$ to $0.04 \%$ more for $\bar{d}=-0.45$ ), although a strong extrapolation rate can improve the profitability of the strategy with lag 26 (at about $5 \%$ to $7 \%$ for $\bar{d}=-2.0$ ).

- The ACs become less significant when agents extrapolate weakly (say, $\bar{d}=-0.45$ ), as indicated in Table A.2.6, and more significant when agent extrapolate strongly (say, $\bar{d}=-2.0$ ).

5.2.1. Summary. In summarizing, we obtain the following results when both types of agents follow the same contrarian trading strategy, but with different memory lengths. 

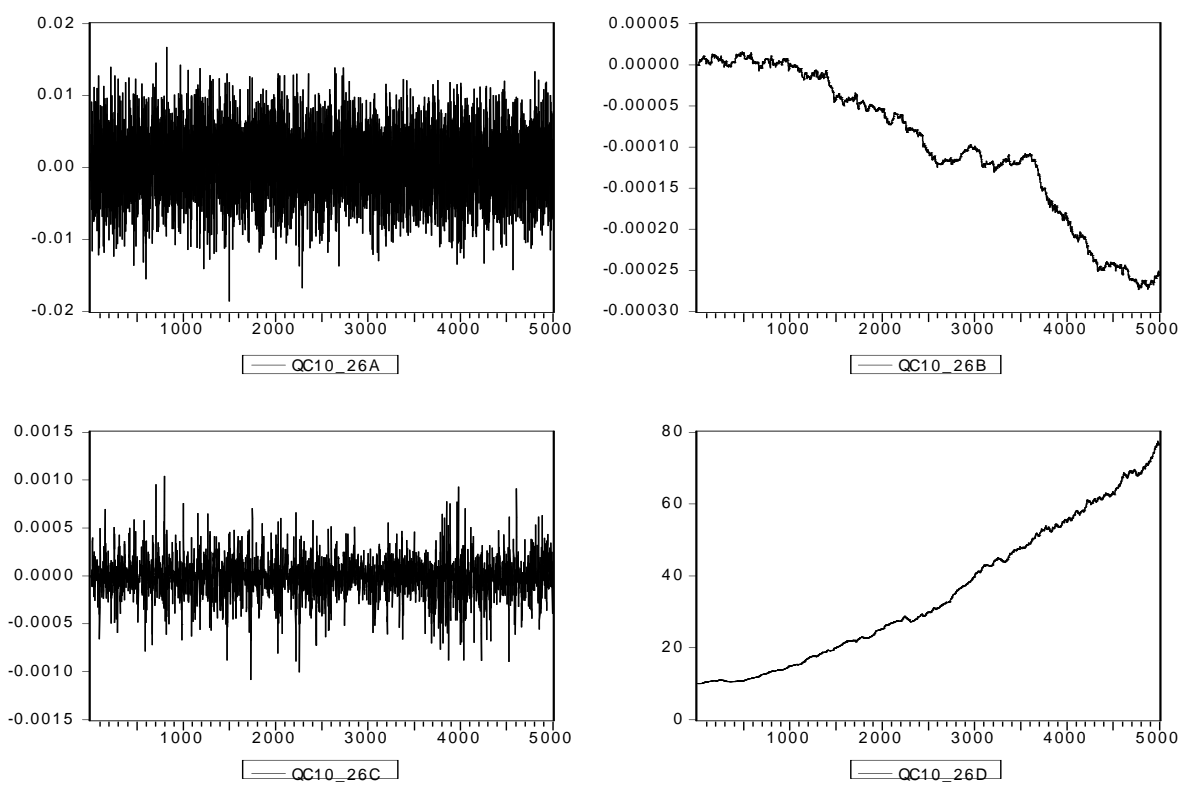

FIGURE 5.3. Time series plots for wealth and population distributions, returns and prices when the same contrarian trading strategies with different lags $\left(L_{1}, L_{2}\right)=(10,26)$ are used. Here, $\bar{\delta}=0.5, \bar{d}=$ -0.4 and $q=0.01$.

- Without the noisy process and a given set of parameters, an increase in lag length of the trading strategies stabilizes the return series of the underlying deterministic system. As both $\bar{d}$ and $\beta$ are near their bifurcation values, profitability of trading strategies and herd behavior are observed, in general.

- Adding a noisy dividend yield process, in general, improves the profitability of the trading strategies with long lag lengths (say, $L_{2}=5,7,26$ ). However, such profitability becomes less significant when the relative difference between the two lag lengths is small (say, $L_{1}=10, L_{2}=14$ ).

- Similar to the case of momentum trading strategies, herd behavior is observed when one of the trading strategies becomes (significantly) profitable. Also, over-concentration (in terms of the initial average wealth and population proportion), or over extrapolation (in terms of low extrapolation rates and improper risk premium levels) can cause overshooting of price and lead to price explosion, and to a market crash. Price levels are more determined by the risk premium levels than by the other parameters (such as extrapolation rate and switching intensity). 
- The ACs are significantly negative for odd lags and positive for even lags when the memory lengths of the contrarian trading strategies are small. However, they become less significant when the memory lengths increase.

\section{CONCLUSIONS}

This paper has taken the basic two-date portfolio optimization model that is the basis of asset pricing theories (such as CAPM) and adds into it investor heterogeneity by allowing agents to form different views on the expected value of the return distribution of the risky asset. The outcome is an adaptive model of asset price and wealth dynamics with agents using various trading strategies. As a special case, a quasi-homogeneous model of two types of agents using either momentum or contrarian trading strategies is introduced to analyze the profitability of the trading strategies over different time intervals. It is found that agents with different time-horizons coexist. Our results shed light on the empirical finding that momentum trading strategies are more profitable over short time intervals, while contrarian trading strategies are more profitable over long time intervals. It should be pointed out that this is an unexpected result given the traditional foundations of the adaptive model. Even though the quasi-homogeneous model is one of the simplest cases of the adaptive model, it generates various phenomena observed in financial markets, including rational adaptiveness of agents, overconfidence and underreaction, overreaction and price overshooting, herd behavior, excess volatility, and volatility clustering. The model also displays the essential characteristics of the standard asset price dynamics model assumed in continuous time finance in that the asset price is fluctuating around a geometrically growing trend.

Our analysis in this paper is based on a simplified quasi-homogeneous model further analysis of which is contained in Chiarella, Dieci and Gardini (2006). A more extensive analysis of the adaptive model is necessary in order to explore the potential explanatory power of the model. One of the extensions is to consider models of two or three different types of trading strategies, to analyze the profitability of different trading strategies, and to examine the stylized facts of the return distribution. Secondly, the attitudes of agents towards the extrapolation and risk premium change when the market environment changes and this change should be made endogenous. Thirdly, there should be a more extensive simulation study of these richer models once they are developed. In fact a proper Monte-Carlo analysis is required to determine whether the models can generate with a high frequency the statistical characteristics of major indices such as the S\&P500. These extensions are interesting problems which are left to future research work. 
Appendix A.1. Proof of Proposition 2.1

Proof. At time period $t-1$, assume that there are $\ell_{j, t-1}$ agents belonging to group $j$. Then, within the group, their optimum demand on wealth proportion to be invested in the risky asset are the same, denoted by $\bar{\pi}_{j, t-1}$. It follows from (2.2) and (2.7) that their average wealth proportion, which corresponds to the wealth proportion of the $j$-th HRA, at the next time period $t$ is given by

$$
\begin{aligned}
\bar{w}_{j, t} & =\frac{\bar{W}_{j, t}}{\bar{W}_{t}}=\frac{\bar{W}_{j, t-1}\left[R+\left(\rho_{t}-r\right) \bar{\pi}_{j, t-1}\right]}{\bar{W}_{t}} \\
& =\frac{\bar{w}_{j, t-1}\left[R+\left(\rho_{t}-r\right) \bar{\pi}_{j, t-1}\right]}{\bar{W}_{t} / \bar{W}_{t-1}} .
\end{aligned}
$$

Note that

$$
\frac{\bar{W}_{t}}{\bar{W}_{t-1}}=\frac{\sum_{j=1}^{h} \bar{W}_{j, t}}{\bar{W}_{t-1}}=\sum_{k=1}^{h} \bar{w}_{k, t-1}\left[R+\left(\rho_{t}-r\right) \bar{\pi}_{k, t-1}\right] .
$$

Then both (A.1.1) and (A.1.2) imply that the time $t$ average wealth proportion for the $j$-th group, formed at $t-1$, is given by (2.9).

With the notations introduced in Section 2, the market clearing equilibrium price equation (2.6) can be rewritten as:

$$
\sum_{j=1}^{h} n_{j, t} \bar{\pi}_{j, t} \bar{W}_{j, t}=N p_{t} / H
$$

Note that

$$
W_{t}=\sum_{j=1}^{H} W_{j, t}=\sum_{j=1}^{h} \ell_{j, t} \bar{W}_{j, t}=H \sum_{j=1}^{h} n_{j, t} \bar{W}_{j, t} .
$$

It follows from (A.1.3) and (A.1.4) that the market clearing price equilibrium equation (A.1.3) becomes

$$
W_{t} \sum_{j=1}^{h} n_{j, t} \bar{\pi}_{j, t} \bar{w}_{j, t}=N p_{t} \sum_{j=1}^{h} n_{j, t} \bar{w}_{j, t}
$$

From (A.1.5)

$$
\frac{W_{t}}{W_{t-1}} \frac{\sum_{j=1}^{h} n_{j, t} \bar{\pi}_{j, t} \bar{w}_{j, t}}{\sum_{j=1}^{h} n_{j, t-1} \bar{\pi}_{j, t-1} \bar{w}_{j, t-1}}=\left(1+\rho_{t}-\alpha_{t}\right) \frac{\sum_{j=1}^{h} n_{j, t} \bar{w}_{j, t}}{\sum_{j=1}^{h} n_{j, t-1} \bar{w}_{j, t-1}} .
$$

Note that

$$
\frac{W_{t}}{W_{t-1}}=\frac{\sum_{j=1}^{h} n_{j, t} \bar{W}_{j, t}}{\sum_{j=1}^{h} n_{j, t-1} \bar{W}_{j, t-1}}=\frac{\sum_{j=1}^{h} n_{j, t} \bar{w}_{j, t-1}\left[R+\left(\rho_{t}-r\right) \bar{\pi}_{j, t-1}\right]}{\sum_{j=1}^{h} n_{j, t-1} \bar{w}_{j, t-1}} .
$$

Substituting (A.1.7) into (A.1.6),

$$
\begin{aligned}
\sum_{j=1}^{h} n_{j, t} \bar{w}_{j, t-1}\left[R+\left(\rho_{t}-r\right) \bar{\pi}_{j, t-1}\right] \sum_{j=1}^{h} n_{j, t} \bar{\pi}_{j, t} \bar{w}_{j, t} \\
=\left(1+\rho_{t}-\alpha_{t}\right) \sum_{j=1}^{h} n_{j, t} \bar{w}_{j, t} \sum_{j=1}^{h} n_{j, t-1} \bar{\pi}_{j, t-1} \bar{w}_{j, t-1} .
\end{aligned}
$$


Also, using (2.9),

$$
\begin{aligned}
\sum_{j=1}^{h} n_{j, t} \bar{\pi}_{j, t} \bar{w}_{j, t} & =\frac{\sum_{j=1}^{h} n_{j, t} \bar{\pi}_{j, t} \bar{w}_{j, t-1}\left[R+\left(\rho_{t}-r\right) \bar{\pi}_{j, t-1}\right]}{\sum_{k=1}^{h} \bar{w}_{k, t-1}\left[R+\left(\rho_{t}-r\right) \bar{\pi}_{k, t-1}\right]}, \\
\sum_{j=1}^{h} n_{j, t} \bar{w}_{j, t} & =\frac{\sum_{j=1}^{h} n_{j, t} \bar{w}_{j, t-1}\left[R+\left(\rho_{t}-r\right) \bar{\pi}_{j, t-1}\right]}{\sum_{k=1}^{h} \bar{w}_{k, t-1}\left[R+\left(\rho_{t}-r\right) \bar{\pi}_{k, t-1}\right]} .
\end{aligned}
$$

Substitution of (A.1.9) and (A.1.10) into (A.1.8) and simplification of the corresponding expression leads to equation

$$
\begin{aligned}
& \sum_{j=1}^{h} n_{j, t} \bar{w}_{j, t-1} \bar{\pi}_{j, t}\left[R+\left(\rho_{t}-r\right) \bar{\pi}_{j, t-1}\right] \\
&=\left[\left(\rho_{t}-r\right)+\left(1+r-\alpha_{t}\right)\right]\left(\sum_{j=1}^{h} n_{j, t-1} \bar{\pi}_{j, t-1} \bar{w}_{j, t-1}\right) .
\end{aligned}
$$

Solving for $\rho_{t}$ from (A.1.11), one obtains equation (2.10) for the return $\rho_{t}$. 
Appendix A.2. TIME SERIES PlOtS, STATISTIC AND AUTOCORRELATION RESUlTS

For both momentum and contrarian trading strategies with different combinations of lag lengths $\left(L_{1}, L_{2}\right)$, this appendix provide

- Time series plots for wealth ( $\bar{w}_{t}$, the difference of wealth proportions), population $\left(n_{t}\right.$, the difference of population proportions), returns $\left(\rho_{t}\right)$, and prices $\left(p_{t}\right)$;

- Numerical comparative statics for wealth (WEA), population (POP), and returns (RET);

- Autocorrelation coefficients (AC) for return series with lags from 1 to 36.
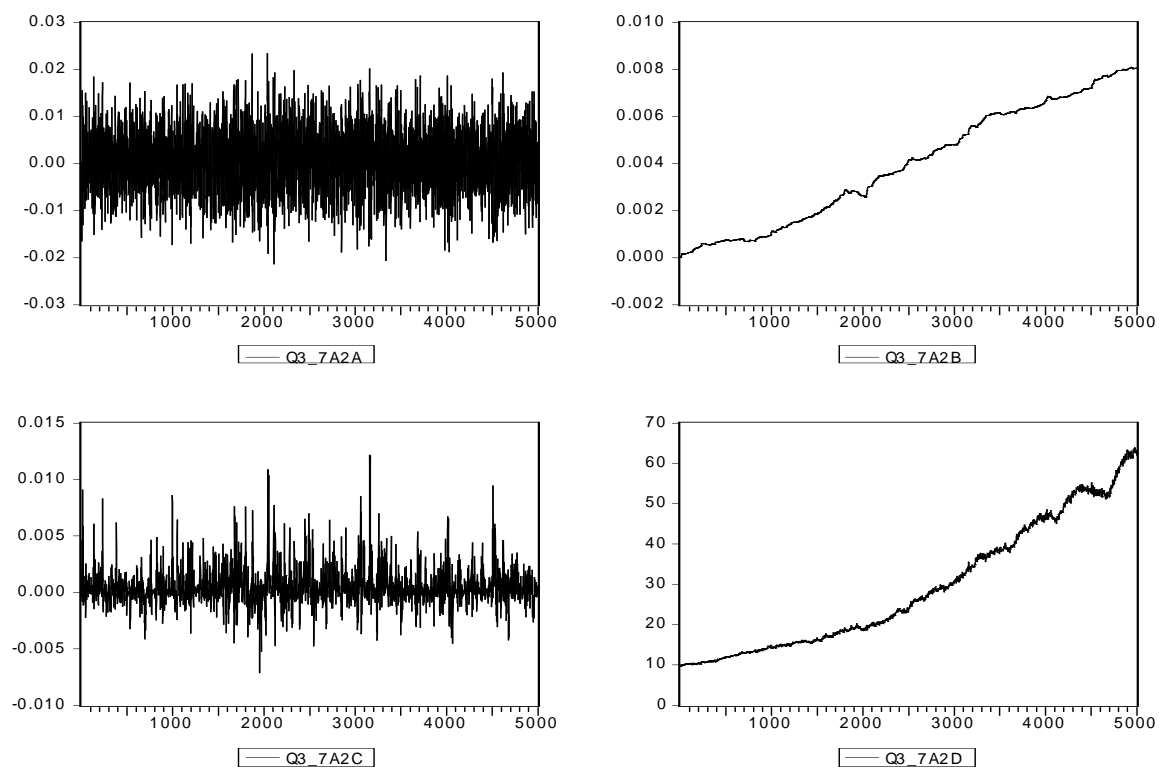

FIgURE A.2.1. Time series plots of wealth and population distributions, returns and prices when the same momentum trading strategies with different lags $\left(L_{1}, L_{2}\right)=(3,7)$ are used. Here, $\bar{\delta}=0.6, \bar{d}=0.53$ and $q=0.03$. 

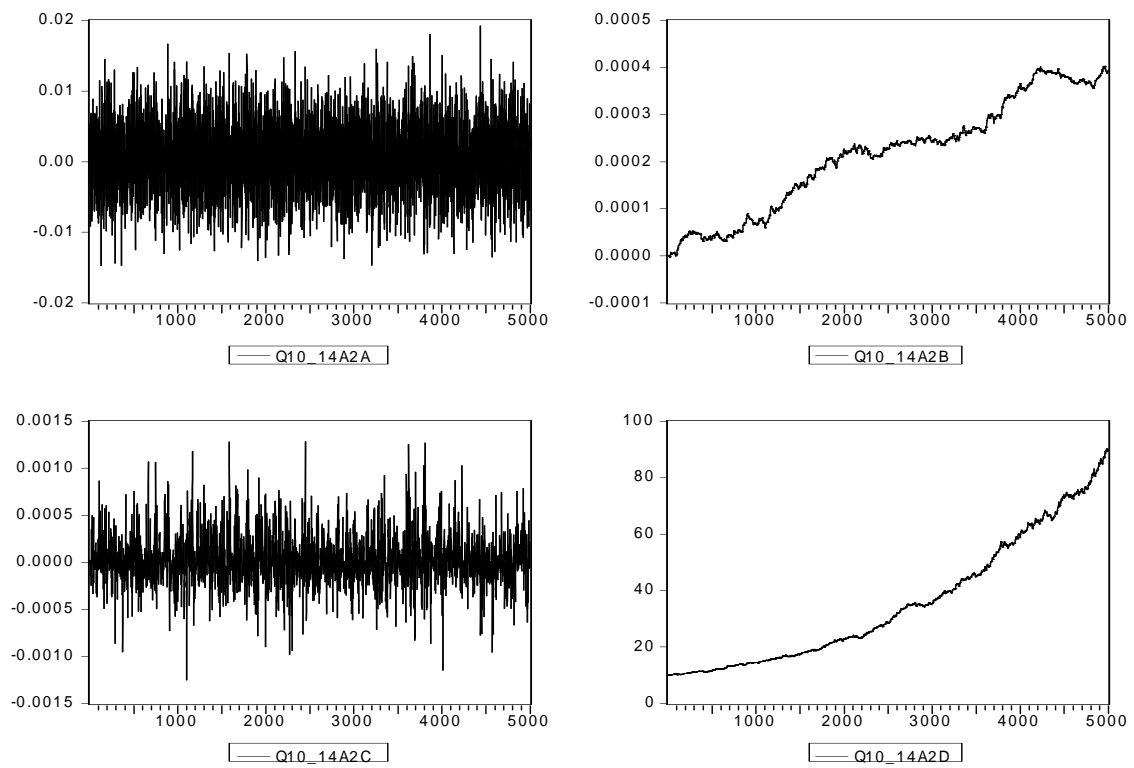

FIgURE A.2.2. Time series plots of wealth and population distributions, returns and prices when the same momentum trading strategies with different lags $\left(L_{1}, L_{2}\right)=(10,14)$ are used. Here, $\bar{\delta}=0.6, \bar{d}=$ 0.53 and $q=0.03$.

\begin{tabular}{|c|c|c|c|c|c|c|}
\hline & & $(3,5)$ & & & $(3,7)$ & \\
\hline & RET & WEA & POP & RET & WEA & POP \\
\hline Mean & 0.000721 & 0.023248 & 0.003062 & 0.000525 & 0.003973 & 0.000484 \\
\hline Median & 0.000691 & 0.022135 & 0.001505 & 0.000542 & 0.004138 & 0.000219 \\
\hline Maximum & 0.043964 & 0.050955 & 0.042773 & 0.023386 & 0.008098 & 0.012162 \\
\hline Minimum & -0.046662 & $-1.00 \mathrm{E}-06$ & -0.002759 & -0.021407 & $-4.00 \mathrm{E}-06$ & -0.00715 \\
\hline Std. Dev. & 0.011343 & 0.014838 & 0.004475 & 0.006339 & 0.002526 & 0.001512 \\
\hline Skewness & -0.020851 & 0.155153 & 2.998613 & 0.023471 & 0.032782 & 1.6045 \\
\hline Kurtosis & 3.241269 & 1.815871 & 15.84896 & 2.9332 & 1.589621 & 10.42554 \\
\hline Jarque-Bera & 12.49201 & 312.2396 & 41896.41 & 1.389 & 415.389 & 13635.28 \\
\hline Probability & 0.001938 & 0 & 0 & 0.499324 & 0 & 0 \\
\hline
\end{tabular}

TABLE A.2.1. Statistics of time series of wealth, population and returns for momentum trading strategies with $\left(L_{1}, L_{2}\right)=(3,5),(3,7)$ and $\bar{\delta}=0.6, \bar{d}=0.53$ and $q=0.03$. 

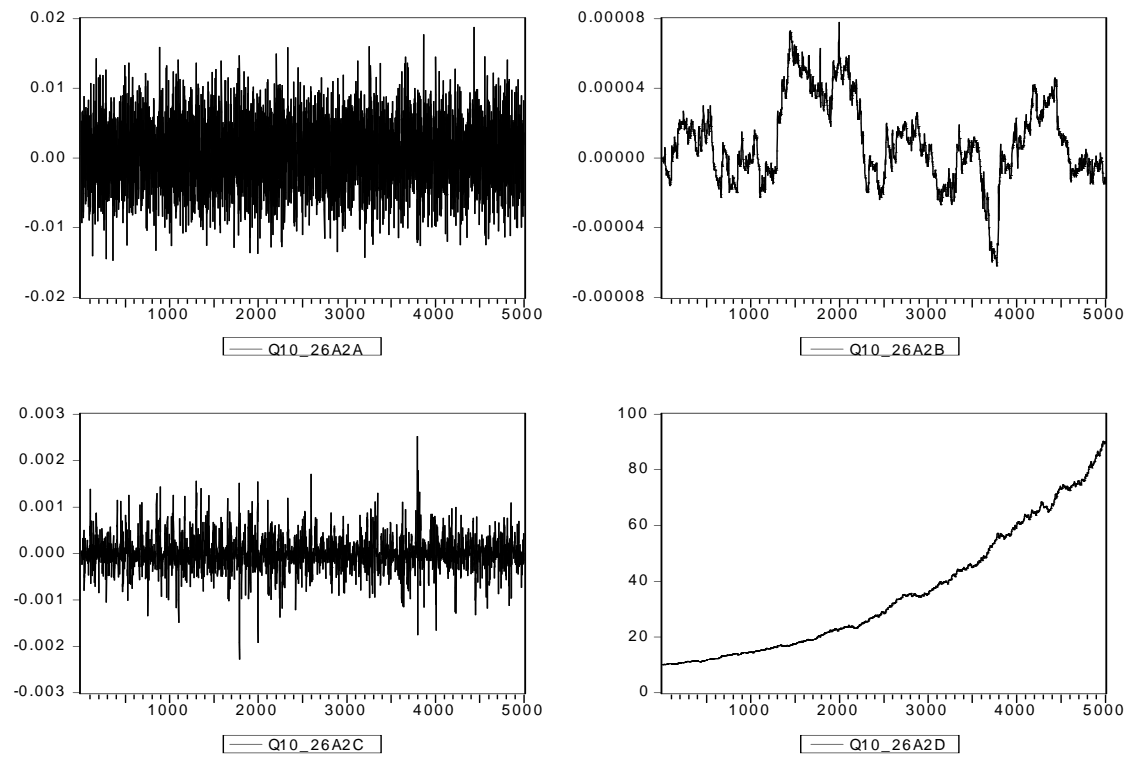

FIgURE A.2.3. Time series plots of wealth and population distributions, returns and prices when the same momentum trading strategies with different lags $\left(L_{1}, L_{2}\right)=(10,26)$ are used. Here, $\bar{\delta}=0.6, \bar{d}=$ 0.53 and $q=0.03$.

\begin{tabular}{|c|c|c|c|c|c|c|}
\hline & & $(10,14)(a)$ & & & $(10,14)(b)$ & \\
\hline & RET & WEA & POP & RET & WEA & POP \\
\hline Mean & 0.000641 & 0.000215 & $2.36 \mathrm{E}-05$ & 0.000739 & 0.004879 & 0.000536 \\
\hline Median & 0.000682 & 0.000233 & $6.00 \mathrm{E}-06$ & 0.000685 & 0.005311 & 0.000173 \\
\hline Maximum & 0.019241 & 0.000403 & 0.001285 & 0.02642 & 0.008898 & 0.015336 \\
\hline Minimum & -0.014757 & $-3.00 \mathrm{E}-06$ & -0.001252 & -0.021672 & 0 & -0.004178 \\
\hline Std. Dev. & 0.004793 & 0.000118 & 0.000232 & 0.006001 & 0.002481 & 0.001424 \\
\hline Skewness & -0.016961 & $-1.52 \mathrm{E}-01$ & 0.506932 & 0.069921 & -0.211947 & 2.629638 \\
\hline Kurtosis & 3.018244 & 1.900675 & 6.319931 & 3.119651 & 2.01417 & 15.73849 \\
\hline Jarque-Bera & 0.309123 & 270.9888 & 2510.89 & 7.058159 & 239.9537 & 39576.5 \\
\hline Probability & 0.856791 & 0 & 0 & 0.029332 & 0 & 0 \\
\hline
\end{tabular}

TABLE A.2.2. Statistics of time series of wealth, population and returns for momentum trading strategies with $\left(L_{1}, L_{2}\right)=(10,14)$ and $\bar{\delta}=0.6, q=0.03, \bar{d}=0.53$ for (a) and $\bar{d}=1.2$ for (b). 


\begin{tabular}{|c|c|c|c|c|c|c|c|}
\hline & & $(10,26)(\mathrm{a})$ & & & & $(10,26)(\mathrm{b})$ & \\
\hline & RET & WEA & POP & RET & WEA & POP \\
\hline Mean & 0.000641 & $8.66 \mathrm{E}-06$ & $-7.90 \mathrm{E}-07$ & & 0.000651 & -0.000483 & $-6.68 \mathrm{E}-05$ \\
Median & 0.000678 & $5.00 \mathrm{E}-06$ & $-1.30 \mathrm{E}-05$ & & 0.000521 & -0.000365 & $-8.70 \mathrm{E}-05$ \\
Maximum & 0.01877 & $7.80 \mathrm{E}-05$ & 0.002522 & & 0.020166 & 0.000299 & 0.008564 \\
Minimum & $-1.48 \mathrm{E}-02$ & $-6.20 \mathrm{E}-05$ & -0.002285 & & -0.019343 & -0.001257 & -0.007335 \\
Std. Dev. & 0.00473 & $2.33 \mathrm{E}-05$ & 0.000328 & & 0.005295 & 0.000433 & 0.001248 \\
Skewness & $-2.04 \mathrm{E}-02$ & 0.217143 & 0.159003 & & 0.090161 & -0.091597 & 0.23871 \\
Kurtosis & 3.001559 & 3.132038 & 7.858779 & & 2.949879 & 1.620711 & 7.002653 \\
\hline Jarque-Bera & 0.346695 & 42.93333 & 4940.334 & 7.298971 & 403.4138 & 3385.919 \\
Probability & 0.840845 & 0 & 0 & & 0.026004 & 0 & 0 \\
\hline
\end{tabular}

TABLE A.2.3. Statistics of time series of wealth, population and returns for momentum trading strategies with $\left(L_{1}, L_{2}\right)=(10,26)$, $q=0.03$, and $\bar{\delta}=0.6, \bar{d}=0.53$ for (a), and $\bar{\delta}=0.6, \bar{d}=1.2$ for (b).
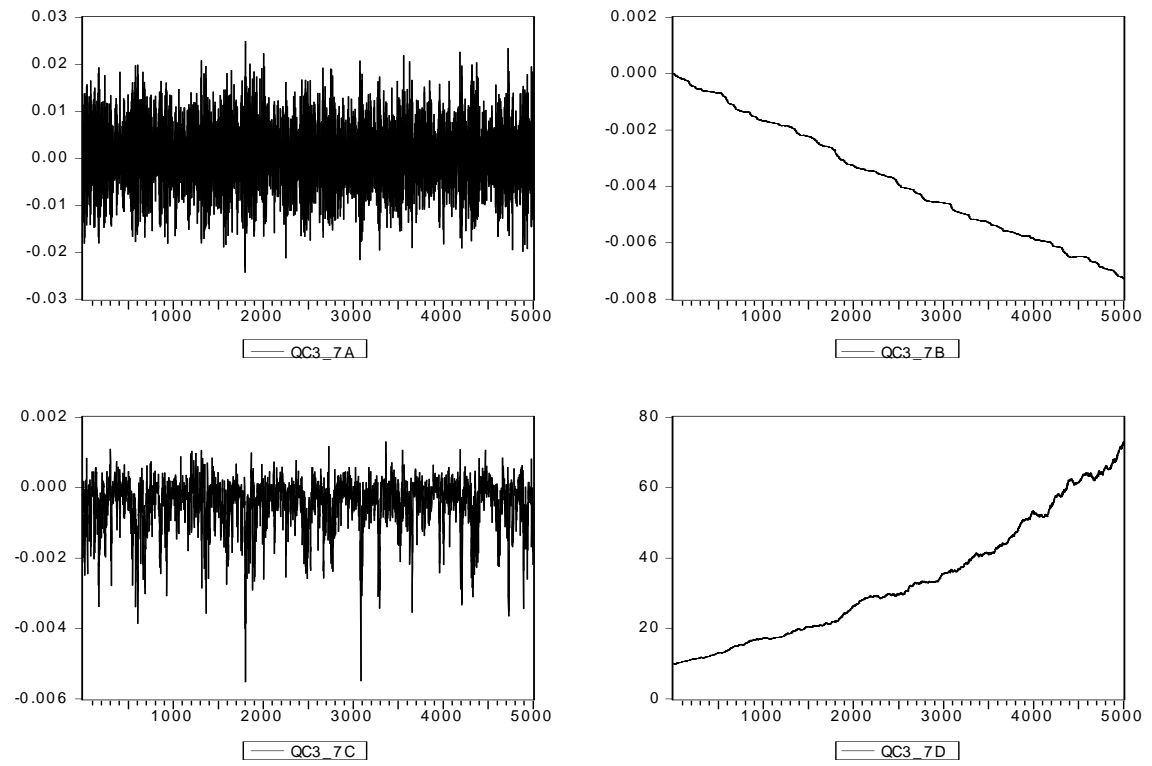

FIguRE A.2.4. Time series plots of wealth and population distributions, returns and prices when the same contrarian trading strategies with different lags $\left(L_{1}, L_{2}\right)=(3,7)$ are used. Here, $\bar{\delta}=0.6, \bar{d}=$ -0.45 and $q=0.03$. 


\begin{tabular}{|r|r|r|r|r|r|r|}
\hline Lag & $(3,5)$ & $(3,7)$ & $(10,14)$ & $(10,14)(\mathrm{a})$ & $(10,26)$ & $(10,26)(\mathrm{a})$ \\
\hline \hline 1 & 0.775 & 0.567 & 0.21 & 0.519 & 0.17 & 0.369 \\
2 & 0.412 & 0.265 & 0.063 & 0.293 & 0.048 & 0.157 \\
3 & -0.04 & 0.009 & 0.025 & 0.178 & 0.019 & 0.076 \\
4 & -0.492 & -0.317 & -0.015 & 0.146 & -0.023 & 0.041 \\
5 & -0.753 & -0.366 & -0.018 & 0.105 & -0.021 & 0.025 \\
6 & -0.824 & -0.314 & 0.006 & 0.06 & 0.006 & -0.016 \\
7 & -0.643 & -0.257 & 0.01 & 0.005 & 0.01 & -0.03 \\
8 & -0.287 & -0.182 & -0.009 & -0.036 & -0.009 & -0.048 \\
9 & 0.118 & -0.057 & 0.002 & -0.1 & 0.005 & -0.075 \\
10 & 0.489 & 0.037 & -0.017 & -0.191 & -0.013 & -0.113 \\
11 & 0.696 & 0.108 & -0.095 & -0.35 & -0.094 & -0.284 \\
12 & 0.704 & 0.189 & -0.044 & -0.308 & -0.033 & -0.202 \\
13 & 0.514 & 0.185 & -0.016 & -0.258 & -0.005 & -0.094 \\
14 & 0.187 & 0.135 & -0.026 & -0.239 & -0.005 & -0.037 \\
15 & -0.176 & 0.084 & -0.114 & -0.304 & -0.029 & -0.009 \\
16 & -0.478 & -0.007 & -0.041 & -0.245 & -0.002 & 0.017 \\
17 & -0.628 & -0.065 & -0.016 & -0.167 & 0 & 0.034 \\
18 & -0.602 & -0.077 & -0.007 & -0.106 & -0.002 & 0.03 \\
19 & -0.411 & -0.084 & 0.019 & -0.05 & 0.017 & 0.028 \\
20 & -0.114 & -0.084 & 0.015 & -0.028 & 0.013 & 0.035 \\
21 & 0.2 & -0.057 & -0.009 & 0.023 & -0.012 & 0.045 \\
22 & 0.448 & -0.033 & -0.007 & 0.09 & -0.006 & 0.066 \\
23 & 0.56 & -0.022 & -0.002 & 0.107 & -0.002 & 0.063 \\
24 & 0.509 & 0.018 & -0.014 & 0.12 & -0.017 & 0.053 \\
25 & 0.322 & 0.038 & 0.013 & 0.15 & 0.009 & 0.014 \\
26 & 0.05 & 0.04 & 0.031 & 0.191 & 0.006 & -0.019 \\
27 & -0.227 & 0.065 & 0.018 & 0.192 & -0.036 & -0.106 \\
28 & -0.424 & 0.048 & 0.017 & 0.172 & -0.001 & -0.091 \\
29 & -0.499 & 0.018 & -0.002 & 0.147 & -0.011 & -0.055 \\
30 & -0.428 & 0 & 0.014 & 0.115 & 0.001 & -0.03 \\
31 & -0.241 & -0.029 & 0.003 & 0.099 & -0.004 & -0.008 \\
32 & 0.006 & -0.044 & 0.036 & 0.054 & 0.034 & 0.008 \\
33 & 0.241 & -0.038 & 0.014 & 0.027 & 0.015 & 0.007 \\
34 & 0.399 & -0.033 & -0.032 & -0.003 & -0.03 & 0.008 \\
35 & 0.436 & -0.03 & -0.014 & -0.016 & -0.011 & -0.015 \\
36 & 0.348 & 0.001 & -0.023 & -0.041 & -0.023 & 0.011 \\
\hline
\end{tabular}

TABLE A.2.4. Autocorrelation coefficients (AC) of returns for momentum trading strategies with $\left(L_{1}, L_{2}\right)=(3,5),(3,7),(10,14)$ and $(10,26)$. The parameters are: $\bar{\delta}=0.6, \bar{d}=0.53$ and $q=0.03$ for $(3,5),(3,7),(10,14)$ and $(10,26) ; \bar{\delta}=0.6, \bar{d}=1.2$ and $q=0.03$ for $(10,14)(a)$; and $\bar{\delta}=0.6, \bar{d}=1.2$ and $q=0.03$ and $(10,26)(a)$. 


\begin{tabular}{|c|c|c|c|c|c|c|}
\hline & & $(3,5)$ & & & $(3,7)$ & \\
\hline & RET & WEA & POP & RET & WEA & POP \\
\hline Mean & 0.000732 & -0.021357 & -0.002489 & 0.000586 & -0.003781 & -0.000436 \\
Median & 0.000565 & -0.020961 & -0.00115 & 0.00061 & -0.003947 & -0.000253 \\
Maximum & 0.058371 & $1.00 \mathrm{E}-06$ & 0.001417 & 0.024943 & $1.00 \mathrm{E}-06$ & 0.001301 \\
Minimum & -0.049512 & -0.041469 & -0.02122 & -0.024361 & -0.007294 & -0.005522 \\
Std. Dev. & 0.0172 & 0.012664 & 0.003256 & 0.007258 & 0.002071 & 0.000648 \\
Skewness & $3.94 \mathrm{E}-02$ & -0.23075 & -1.79741 & $-2.38 \mathrm{E}-02$ & 0.128596 & -1.706059 \\
Kurtosis & 2.623585 & 1.776767 & 6.184726 & 2.73569 & 1.77624 & 7.727226 \\
\hline Jarque-Bera & 30.8166 & 356.1715 & 4806.214 & 15.02887 & 325.8435 & 7082.505 \\
Probability & 0 & 0 & 0 & 0.000545 & 0 & 0 \\
\hline \hline & & $(10,14)$ & & & $(10,26)$ & \\
\hline & RET & WEA & POP & RET & WEA & POP \\
\hline Mean & 0.000546 & $-1.36 \mathrm{E}-05$ & $-1.74 \mathrm{E}-06$ & 0.000587 & $-9.89 \mathrm{E}-05$ & $-1.53 \mathrm{E}-05$ \\
Median & 0.000481 & $-5.00 \mathrm{E}-06$ & $-5.00 \mathrm{E}-06$ & 0.000674 & $-1.00 \mathrm{E}-04$ & $-4.00 \mathrm{E}-06$ \\
Maximum & 0.018059 & $2.10 \mathrm{E}-05$ & 0.000912 & 0.016719 & $1.60 \mathrm{E}-05$ & 0.001042 \\
Minimum & -0.018072 & $-5.40 \mathrm{E}-05$ & -0.000972 & -0.018608 & $-2.74 \mathrm{E}-04$ & -0.001083 \\
Std. Dev. & 0.004875 & $2.01 \mathrm{E}-05$ & 0.000136 & 0.004787 & $8.84 \mathrm{E}-05$ & 0.000182 \\
Skewness & 0.067356 & -0.269859 & 0.137827 & -0.084361 & -0.498882 & -0.30024 \\
Kurtosis & 3.073003 & 1.604405 & 7.232651 & 2.928112 & 2.074143 & 6.742584 \\
\hline Jarque-Bera & 4.891911 & 466.5478 & 3748.941 & 7.008679 & 386.0658 & 2993.831 \\
Probability & 0.086643 & 0 & 0 & 0.030067 & 0 & 0 \\
\hline
\end{tabular}

TABLE A.2.5. Statistics of time series of wealth, population and returns for contrarian trading strategies with parameters $\bar{\delta}=0.6, \bar{d}=$ -0.45 and $q=0.03$ and lag length combinations $\left(L_{1}, L_{2}\right)$ are $(3,5)$ for (a), $(3,7)$ for $(b),(10,14)$ for $(c)$, and $(10,26)$ for $(d)$. 


\begin{tabular}{|r|r|r|r|r|}
\hline Lag & $(3,5)$ & $(3,7)$ & $(10,14)$ & $(10,26)$ \\
\hline \hline 1 & -0.944 & -0.678 & -0.144 & -0.155 \\
2 & 0.922 & 0.53 & 0.056 & 0.047 \\
3 & -0.914 & -0.529 & -0.014 & -0.025 \\
4 & 0.93 & 0.636 & -0.01 & 0.003 \\
5 & -0.924 & -0.563 & -0.001 & 0.001 \\
6 & 0.926 & 0.479 & 0 & -0.003 \\
7 & -0.914 & -0.462 & 0.016 & 0.01 \\
8 & 0.909 & 0.533 & -0.003 & 0.004 \\
9 & -0.902 & -0.51 & 0.009 & 0.001 \\
10 & 0.906 & 0.452 & 0.011 & -0.003 \\
11 & -0.9 & -0.428 & 0.097 & 0.107 \\
12 & 0.896 & 0.451 & -0.016 & -0.039 \\
13 & -0.891 & -0.427 & 0.026 & 0.009 \\
14 & 0.889 & 0.393 & -0.013 & 0.006 \\
15 & -0.884 & -0.371 & 0.051 & -0.027 \\
16 & 0.882 & 0.37 & -0.017 & 0.009 \\
17 & -0.876 & -0.366 & 0.014 & -0.004 \\
18 & 0.875 & 0.351 & -0.013 & 0.013 \\
19 & -0.869 & -0.337 & 0.009 & -0.001 \\
20 & 0.867 & 0.34 & -0.008 & -0.01 \\
21 & -0.863 & -0.322 & 0.01 & 0.004 \\
22 & 0.861 & 0.297 & 0.025 & -0.001 \\
23 & -0.858 & -0.282 & -0.008 & -0.016 \\
24 & 0.857 & 0.277 & 0.016 & -0.014 \\
25 & -0.851 & -0.271 & -0.02 & 0 \\
26 & 0.849 & 0.245 & 0.012 & -0.002 \\
27 & -0.844 & -0.232 & -0.002 & 0.043 \\
28 & 0.844 & 0.228 & 0.014 & -0.014 \\
29 & -0.841 & -0.224 & -0.001 & 0.021 \\
30 & 0.838 & 0.215 & -0.002 & -0.019 \\
31 & -0.833 & -0.202 & -0.019 & 0.013 \\
32 & 0.83 & 0.193 & -0.01 & -0.009 \\
33 & -0.828 & -0.186 & -0.012 & 0.007 \\
34 & 0.825 & 0.181 & 0.021 & 0.001 \\
35 & -0.82 & -0.162 & 0.01 & 0.008 \\
36 & 0.817 & 0.162 & 0.001 & 0.01 \\
\hline
\end{tabular}

TABLE A.2.6. Autocorrelation coefficients (AC) of returns for contrarian trading strategies with parameters $\bar{\delta}=0.6, \bar{d}=-0.45, \beta=$ 0.5 and $q=0.03$ and lag length combinations $\left(L_{1}, L_{2}\right)=$ $(3,5),(3,7),(10,14)$ and $(10,26)$. 


\section{REFERENCES}

Anderson, S. and A. de Palma and J. Thisse (1993), Discrete Choice Theory of Product Differentiation, MIT Press, Cambridge, MA.

Arshanapali, B., Coggin, D. and Doukas, J. (1998), 'Multifactor asset pricing analysis of international value investment strategies', Journal of Portfolio Management 24(4), 10-23.

Asness, C. (1997), 'The interaction of value and momentum strategies', Financial Analysts Journal 53, 29-36.

Barberis, N., Shleifer, A. and Vishny, R. (1998), 'A model of investor sentiment', Journal of Financial Economics 49, 307-343.

Brock, W. and Hommes, C. (1997), 'A rational route to randomness', Econometrica 65, 1059-1095.

Brock, W. and Hommes, C. (1998), 'Heterogeneous beliefs and routes to chaos in a simple asset pricing model', Journal of Economic Dynamics and Control 22, 1235-1274.

Bullard, J. and Duffy, J. (1999), 'Using Genetic Algorithms to Model the Evolution of Heterogeneous Beliefs', Computational Economics 13, 41-60.

Capaul, C., Rowley, I. and Sharpe, W. (1993), 'International value and growth stock returns', Financial Analysts Journal 49(July/Aug.), 27-36.

Chiarella, C. (1992), 'The dynamics of speculative behaviour', Annals of Operations Research 37, 101123.

Chiarella, C., Dieci, R. and Gardini, L. (2006), 'Asset price and wealth dynamics in a financial market with heterogeneous agents', Journal of Economic Dynamics and Control 30, 1755 - 1786.

Chiarella, C. and He, X. (2001), 'Asset pricing and wealth dynamics under heterogeneous expectations', Quantitative Finance 1, 509-526.

Chiarella, C. and He, X. (2002), 'Heterogeneous beliefs, risk and learning in a simple asset pricing model', Computational Economics 19, 95-132.

Chiarella, C. and He, X. (2003), 'Heterogeneous beliefs, risk and learning in a simple asset pricing model with a market maker', Macroeconomic Dynamics 7(4), 503-536.

Daniel, K., Hirshleifer, D. and Subrahmanyam, A. (1998), 'A theory of overconfidence, self-attribution, and security market under- and over-reactions', Journal of Finance 53, 1839-1885.

Day, R. and Huang, W. (1990), 'Bulls, bears and market sheep', Journal of Economic Behavior and Organization 14, 299-329.

Fama, E. and French, K. (1998), 'Value versus growth: The international evidence', Journal of Finance 53, 1975-1999.

Farmer, J. (1999), 'Physicists attempt to scale the ivory towers of finance', Computing in Science and Engineering 1, 26-39.

Farmer, J. and Lo, A. (1999), 'Frontier of finance: Evolution and efficient markets', Proceedings of the National Academy of Sciences 96, 9991-9992.

Franke, R. and Nesemann, T. (1999), 'Two destabilizing strategies may be jointly stabilizing', Journal of Economics 69, 1-18.

Frankel, F. and Froot, K. (1987), 'Using survey data to test propositions regarding exchange rate expectations', American Economic Review 77, 133-153.

Hirshleifer, D. (2001), 'Investor psychology and asset pricing', Journal of Finance 56, 1533-1597.

Hommes, C. (2001), 'Financial markets as nonlinear adaptive evolutionary systems', Quantitative Finance 1, 149-167.

Hong, H. and Stein, J. (1999), 'A unified theory of underreaction, momentum trading, and overreaction in asset markets', Journal of Finance 54, 2143-2184.

Jegadeesh, N. and Titman, S. (1993), 'Returns to buying winners and selling losers: Implications for stock market efficiency', Journal of Finance 48, 65-91.

Jegadeesh, N. and Titman, S. (2001), 'Profitability of momentum strategies: an evaluation of alternative explanations', Journal of Finance 56, 699-720.

Kirman, A. (1992), 'Whom or what does the representative agent represent?', Journal of Economic Perspectives 6, 117-136.

LeBaron, B. (2000), 'Agent based computational finance: suggested readings and early research', Journal of Economic Dynamics and Control 24, 679-702.

Lee, C. and Swaminathan, B. (2000), 'Price momentum and trading volume', Journal of Finance 55, 2017-2069. 
Levis, M. and Liodakis, M. (2001), 'Contrarian strategies and investor expectations: the u.k. evidence', Financial Analysts Journal 57(Sep./Oct.), 43-56.

Levy, M. and Levy, H. (1996), 'The danger of assuming homogeneous expectations', Financial Analysts Journal 52(3), 65-70.

Levy, M., Levy, H. and Solomon, S. (1994), 'A microscopic model of the stock market', Economics Letters 45, 103-111.

Levy, M., Levy, H. and Solomon, S. (2000), Microscopic Simulation of Financial Markets, from investor behavior to market phenomena, Acadmic Press, Sydney.

Lintner, J. (1965), 'The valuation of risk assets and the selection of risky investments in stock portfolios and capital budgets', Review of Economics and Statistics 47, 13-37.

Lux, T. (1995), 'Herd behaviour, bubbles and crashes', Economic Journal 105, 881-896.

Lux, T. and Marchesi, M. (1999), 'Scaling and criticality in a stochastic multi-agent model of a financial markets', Nature 397(11), 498-500.

Manski, C. and McFadden, D. (1981), Structural Analysis of Discrete Data with Econometric Applications, MIT Press.

Merton, R. (1973), 'An intertemporal capital asset pricing model', Econometrica 41, 867-887.

Moskowitz, T. and Grinblatt, M. (1999), 'Do industries explain momentum?', Journal of Finance 54, 1249-1290.

Ross, S. (1976), 'The arbitrage theory of capital asset pricing', Journal of Economic Theory 13, 341360.

Rouwenhorst, K. G. (1998), 'International momentum strategies', Journal of Finance 53, 267-284.

Sharpe, W. (1964), 'Capital asset prices: A theory of market equilibrium under conditions of risk', Journal of Finance 19, 425-442. 